\title{
Restinga de Praia das Neves, ES, Brasil: caracterização fitofisionômica, florística e conservação
}

\author{
Denise Monte Braz', Eliane de Lima Jacques ${ }^{1}$, Genise Vieira Somner ${ }^{1,3}$, Lana da Silva Sylvestre ${ }^{2}$, \\ Maria Mercedes Teixeira da Rosa ${ }^{1}$, Maria Verônica Leite Pereira-Moura ${ }^{1}$, Pedro Germano Filho ${ }^{1}$, \\ Arthur Vinícius dos Santos Couto ${ }^{I}$ \& Thiago Azevedo Amorim ${ }^{I}$ \\ ${ }^{1}$ Departamento de Botânica, Universidade Federal Rural do Rio de Janeiro - UFRRJ, BR 465, BR-465, \\ $K m$ 7, CP 74542, Campus Universitário, CEP 23890-000, Seropédica, RJ, Brasil. www.ufrrj.br \\ ${ }^{2}$ Departamento de Botânica, Universidade Federal do Rio de Janeiro - UFRJ, Av. Carlos Chagas Filho, \\ 373, Cidade Universitária, CEP 21941-902, Rio de Janeiro, RJ, Brasil.www.biologia.ufrj.br \\ ${ }^{3}$ Autor para correspondência: Genise Vieira Somner, email: somner@ufrrj.br
}

BRAZ, D.M., JACQUES, E.L., SOMNER, G.V., SYLVESTRE, L.S., ROSA, M.M.T., PEREIRA-MOURA, M.V.L., GERMANO FILHO, P., COUTO, A.V.S. \& AMORIM, T.A. Sandy coastal plains (restinga) of Praia das Neves, ES, Brazil: phytophysiognomy characterization, flora and conservation. Biota Neotrop. 13 (3): http://www.biotaneotropica.org.br/v13n3/en/abstract?article+bn02613032013

\begin{abstract}
The sandy coastal plains (restinga) of Praia das Neves occupies a large area (ca. $67 \mathrm{~km}^{2}$ ) in the southeastern state of Espírito Santo ( $21^{\circ} 14^{\prime} 35.35^{\prime}$ ' S and 4056’48.68” W), bordered by Itabapoana River on the boundary with the state of Rio de Janeiro. The aim of this study was to identify and characterize the different types of plant communities and associated flora on that sandy coastal plain, including the recognition of the habitats related to the endangered lizard Liolaemus lutzae Mertens, 1938. Field trips to characterize the local flora were carried out on specific campaigns, between the years 2008 and 2011. The plants were identified and deposited in the RBR Herbarium of the Department of Botany at the Universidade Federal Rural do Rio de Janeiro. The communities' characterization was based on local observations and specialized bibliography, considering physical aspects of the environment, habit and composition of plant species. The sandy coastal plain of Praia das Neves brings together seven forest, shrub and herbaceous communities, usually distributed in ridges parallels to the shoreline. There have been recorded 167 species of vascular plants, grouped into 58 families, occurring in different communities. The majority of species occurs in a single community, while Allagoptera arenaria (guririda-praia) stands out for occurring in almost all local communities. The restinga of Praia das Neves maintains extensive areas in good environmental conditions, with some species cited in the red list of endangered species in the state of Espírito Santo, and also with important remnants of coastal communities. Although its greatest extension is within a private area, future actions should ensure the preservation of the local restinga, with its different communities and associated biota, according to the current federal legislation.
\end{abstract}

Keywords: Atlantic forest, Southeastern Brazil, sandy ridges, flora, plant communities, endangered species.

BRAZ, D.M., JACQUES, E.L., SOMNER, G.V., SYLVESTRE, L.S., ROSA, M.M.T., PEREIRA-MOURA, M.V.L., GERMANO FILHO, P., COUTO, A.V.S. \& AMORIM, T.A. Restinga de Praia das Neves, ES, Brasil: caracterização fitofisionômica, florística e conservação. Biota Neotrop. 13(3): http://www.biotaneotropica. org.br/v13n3/pt/abstract?article+bn02613032013

Resumo: A restinga de Praia das Neves ocupa uma extensa área $\left(\mathrm{ca} .67 \mathrm{~km}^{2}\right)$ no extremo sul do Estado do Espírito Santo ( $21^{\circ} 14^{\prime} 35.35^{\prime}$ 'S e 4056'48.68”W), limitada pelo Rio Itabapoana, na divisa com o Estado do Rio de Janeiro. $\mathrm{O}$ objetivo desse estudo foi identificar e caracterizar as diferentes formações vegetais dessa restinga e sua flora associada, incluindo o reconhecimento dos habitats relacionados ao lagarto ameaçado de extinção Liolaemus lutzae Mertens, 1938. Excursões para caracterização da flora local foram realizadas em campanhas pontuais, entre os anos de 2008 e 2011, e o material botânico foi identificado e posteriormente depositado no Herbário RBR do Departamento de Botânica da Universidade Federal Rural do Rio de Janeiro. A caracterização das diferentes formações encontradas baseou-se em observações locais e na bibliografia especializada, considerando aspectos físicos do ambiente, o hábito e a composição das espécies vegetais. A vegetação da restinga de Praia das Neves reúne sete formações florestais, arbustivas e herbáceas, distribuídas em geral em faixas paralelas a linha da costa. Foram registradas 167 espécies de plantas vasculares, reunidas em 58 famílias, ocorrendo nas diferentes formações. A grande maioria das espécies estudadas é exclusiva de uma única formação, enquanto que Allagoptera arenaria (guriri-da-praia) se destaca por ocorrer em quase todas as formações locais. A restinga de Praia das Neves mantém extensos trechos em bom estado de conservação, com espécies listadas como ameaçadas para a flora do Estado do Espírito Santo e com importantes remanescentes de formações costeiras no Estado. Embora sua maior extensão esteja contida em área privada, ações futuras devem assegurar a preservação da restinga local, com suas formações vegetais e a biota associada, conforme indica a legislação federal vigente.

Palavras-chave: Floresta Atlântica, Sudeste do Brasil, cordões arenosos, flora, formação vegetal, espécies ameaçadas. 


\section{Introdução}

Restingas apresentam um conjunto bastante diversificado de comunidades biológicas, que reflete a influência das condições do solo, do grau de exposição às brisas marinhas e a iluminação (Sampaio 2005), entre outros fatores. No Brasil, ocorrem ao longo da costa em extensões variadas e podem compor um mosaico de vegetação, reunindo desde áreas florestadas até áreas com vegetação herbácea.

A restinga de Praia das Neves está localizada no extremo sul do Estado do Espírito Santo, na divisa com o Rio de Janeiro. A vegetação natural dessa restinga sofreu alteração antrópica desde o século XVI, durante a época de sua ocupação pelos jesuítas e, posteriormente, passou por períodos de plantio e exploração de mandioca para utilização em indústria local (Lamego 1946). Na mesma época, houve uma intensa exploração de madeira, que servia ao estaleiro local para a manufatura e reparo de embarcações, sendo também escoada para outras cidades (Lamego 1946). Atualmente, além da ocupação com o turismo nos finais de semana e veraneio, uma extensa área da restinga sofreu forte devastação para loteamento e exploração imobiliária, suspensos até o momento. Também é prevista a instalação de um porto de exportação de ferro nessa praia. Apesar das intervenções constantes, a restinga de Praia das Neves ainda detém remanescentes florestais e formações arbustivas abertas de restinga bem preservadas, além de extensas áreas com vegetação em diferentes estágios de regeneração.

As restingas do estado do Espírito Santo tem sido amplamente estudadas (Pereira et al. 1992, Pereira \& Gomes 1993, Pereira \& Assis 2000, Assis et al. 2004, Magnago et al. 2011, entre outros), contudo investigações pretéritas sobre a vegetação de Praia das Neves se restringem ao trabalho de Thomaz \& Monteiro (1993), que incluíram a área na análise da distribuição da comunidade halófila-psamófila por todo o Estado. Já com relação à fauna, além da ocorrência de uma espécie ameaçada de extinção, a lagartixa-de-areia, Liolaemus lutzae Mertens, 1938 (Soares \& Araujo 2008), Rocha et al. (2003) indicaram a ampliação do Corredor Central da Mata Atlântica de forma a incluir a restinga de Praia das Neves, com base na elevada diversidade biológica e riqueza de espécies de quatro grupos de vertebrados.

Estudos sobre o manejo de Liolaemus lutzae, coordenados pelo Professor Alexandre Fernandes Bamberg de Araújo, do Departamento de Biologia Animal da Universidade Federal Rural do Rio de Janeiro (UFRRJ) (Soares \& Araujo 2008), vem permitindo o conhecimento de diferentes aspectos da biota da restinga de Praia das Neves. Tendo em vista o conhecimento do habitat da lagartixa-de-areia, o Departamento de Botânica da UFRRJ realizou estudos de toda a flora local, visando o conhecimento dessa extensa área remanescente de restinga capixaba. Este trabalho teve como objetivo principal a identificação e caracterização das diferentes formações vegetais (fitofisionomias) da restinga de Praia das Neves, com espécies vegetais típicas amostradas. Também foi verificada a ocorrência de espécies ameaçadas de extinção da flora com intuito de reiterar a necessidade de preservação dessa restinga.

\section{Material e Métodos}

A restinga de Praia das Neves, localizada ao sul do Estado do Espírito Santo, no Município de Presidente Kennedy (21 ${ }^{\circ} 14 ’ 35.35$ ”S e $\left.40^{\circ} 56^{\prime} 48.68^{\prime \prime} \mathrm{W}\right)$, é contornada ao sul pelo Rio Itabapoana, onde faz divisa com o Rio de Janeiro, e ao Norte é limitada pela formação Barreiras (Figura 1), que formam um grande arco bordejando os depósitos de areias quartzosas. A restinga ocupa uma área de aproximadamente $12,7 \mathrm{~km}$ de extensão e $67 \mathrm{~km}^{2}$ (4.800ha), margeada por brejos e manguezais em associação com os rios Itabapoana e Marobá, especialmente próximo à foz do primeiro. O clima é Tropical

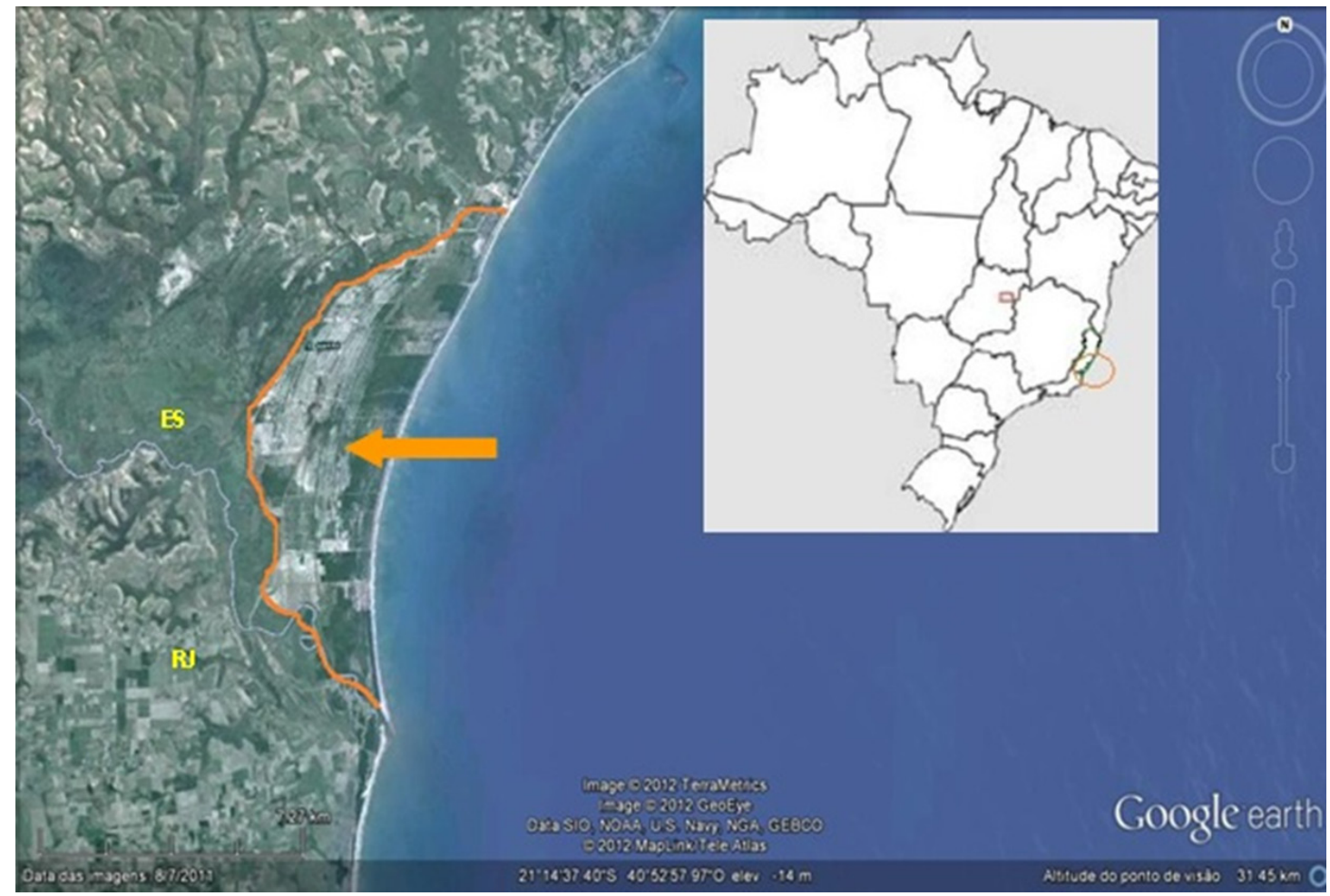

Figura 1. Localização da restinga de Praia das Neves, Município de Presidente Kennedy, ES, Brasil (2114'2408”S e 4058'14.48”O) (Fontes: http://earth. google.com, 2012; http://maps.google.com.br, 1012).

Figure 1. Localization of the sandy coastal plain (restinga) of "Praia das Neves", Municipallty of Presidente Kennedy, ES, Brazil (2114’2408”S and 4058'14.48”O) (Source: http://earth.google.com, 2012; http://maps.google.com.br, 1012). 
(Peel et al. 2007), caracterizado como quente e úmido (três meses secos) de acordo com a classificação de subtipos climáticos regionais do IBGE (Instituto... 2012). Para confecção de mapas e figuras foram utilizadas imagens de satélite de Google Earth (2012).

Foram realizadas nove excursões para coleta de material botânico, assim como para observação dos aspectos físicos, em todas as diferentes formações vegetais da área de estudo, entre abril de 2008 e fevereiro de 2011. O material botânico coletado foi depositado no Herbário do Departamento de Botânica da Universidade Federal Rural do Rio de Janeiro (RBR, sigla segundo Thiers 2013). A identificação do material foi realizada no laboratório através da utilização de literatura especializada, de comparações dos espécimes coletados com os dos acervos dos herbários RBR e do Jardim Botânico do Rio de Janeiro (RB), além do auxílio de especialistas. Foram consideradas 12 espécies registradas apenas através de fotografias, cujas informações de campo foram anotadas e serviram para posterior interpretação e descrição das formações.

A lista de espécies foi organizada por ordem alfabética de família, incluindo informações sobre a ocorrência das mesmas nas diferentes formações vegetais, e seguindo as classificações de Smith et al. (2006), para as samambaias, e do APG III (Angiosperm... 2009), para as Angiospermas. Os nomes das espécies e dos autores foram checados na Lista de espécies da flora do Brasil (Jardim... 2013) e na página do Índex Internacional de Nomes de Plantas (International... 2012).

A distribuição das espécies nos diferentes biomas brasileiros (Amazônia, Caatinga, Cerrado, Floresta Atlântica, Pampas e Pantanal) foi avaliada segundo a Lista de espécies da Flora do Brasil (Jardim... 2013) e nas diferentes formações em bibliografia específica para a Floresta Atlântica (Stehmann et al. 2009).

As fitofisionomias da área de estudo foram reunidas em herbácea, arbustiva e arbórea, com as diferentes formações vegetais locais descritas. Para sua identificação foram utilizadas as classificações de Thomaz \& Monteiro (1993), empregada especificamente para a formação herbácea de praia, denominada halófila-psamófila reptante, sendo as demais formações baseadas em Menezes \& Araújo (2005). Esses autores utilizam alguns termos em comum com Pereira (2003), referentes ao hábito e adensamento da vegetação e na profundidade do lençol freático. Em função do detalhamento e reconhecimento de algumas subdivisões compartilhadas com a área estudada, foi seguida a classificação de Menezes \& Araújo (2005), que igualmente contempla numerosas formações vegetais comuns no sudeste brasileiro. O conceito de "moita" adotado neste trabalho segue a definição proposta por Menezes \& Araújo (2005) - agrupamento com mais de um indivíduo lenhoso, com superposição de copas, separados entre si por areia desnuda ou por espécies herbáceas.

O mapa de vegetação foi elaborado no Laboratório de Herpetologia do Prof. Alexandre F. B. de Araújo, Instituto de Biologia, Universidade Federal Rural do Rio de Janeiro, utilizando como ferramenta o programa Arc GIS 9.3.

\section{Resultados e Discussão}

A Praia das Neves se destaca dos outros trechos de restinga do litoral sul capixaba por apresentar área extensa com diversas formações de restinga preservadas, que variam de formações herbáceas, passando por formações arbustivas e chegando a florestal. Essas formações estão dispostas em dezenas de cordões arenosos paralelos a linha da costa (Figura 2) e entremeadas por áreas alagadas ou alagáveis. A complexidade da vegetação de restinga aumenta a partir da praia em direção ao interior da planície costeira, como foi especificamente detalhado por Silva \& Somner (1984), e amplamente observado nas restingas brasileiras (Amaral et al. 2008).
As linhas de deposição de sedimentos na restinga de Praia das Neves são nítidas (Figura 2, 3a) e muitas vezes marcadas pelo afloramento do lençol freático. Esses afloramentos podem formar brejos, próximo às formações florestais, e encraves inundáveis nos locais mais afastados dos cursos d'água constantes. Da praia para o interior, a vegetação herbácea de praia se adensa, passando a uma vegetação arbustiva e a seguir a florestal (Figura 3b, 3c, 3f, $4 \mathrm{a}, 4 \mathrm{~b}$ ) ou ocorrendo moitas esparsas (Figura 3d, 3e), com encraves de vegetação herbáceo-arbustiva. De acordo com Sampaio (2005), teores de matéria orgânica e de nutrientes do solo, profundidade do lençol freático, salinidade, topografia e mobilidade do substrato são os principais fatores que determinam à fisionomia da vegetação nos diferentes ambientes das restingas.

\section{Fitofisionomias herbáceas}

Halófila-psamófila reptante - Próximo à linha de praia, acima do nível médio das marés altas, ocorre uma vegetação predominantemente constituída por espécies herbáceas reptantes e estoloníferas (Figuras 3c, 4c, 4d). Estende-se por aproximadamente 25 a $150 \mathrm{~m}$ de largura, em praticamente toda a extensão da praia, por cerca de $12 \mathrm{~km}$. Nessa vegetação as plantas são adaptadas a maior incidência de luz solar, maior salinidade e mobilidade do substrato (Sampaio 2005). É o local de ocorrência de Liolaemus luztae, a lagartixa-de-areia, e as espécies vegetais típicas encontradas foram:

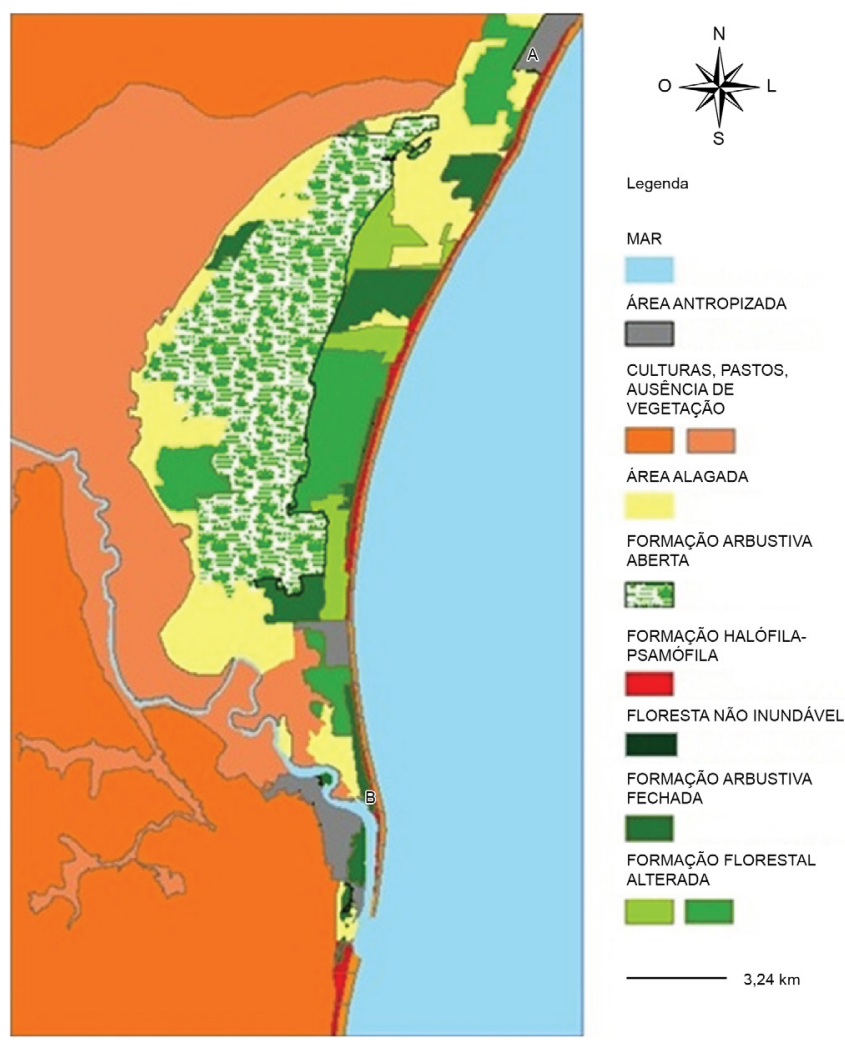

Figura 2. Mapa de vegetação da restinga de Praia das Neves, Município de Presidente Keneddy, ES, Brasil, evidenciando suas principais fitofisionomias

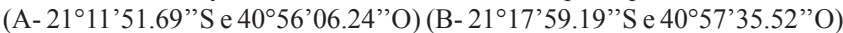
(Google Earth, 2012).

Figure 2. Vegetation map of the sandy coastal plain (restinga) of "Praia das Neves", Municipallty of Presidente Keneddy, ES, Brazil, showing its main phytophysiognomies (A- $21^{\circ} 11^{\prime} 51.69^{\prime \prime} \mathrm{S}$ and $40^{\circ} 56^{\prime} 06.244^{\prime \prime} \mathrm{O}$ ) (B$21^{\circ} 17^{\prime} 59.19^{\prime \prime} \mathrm{S}$ and 4057'35.52'O) (Google Earth, 2012). 

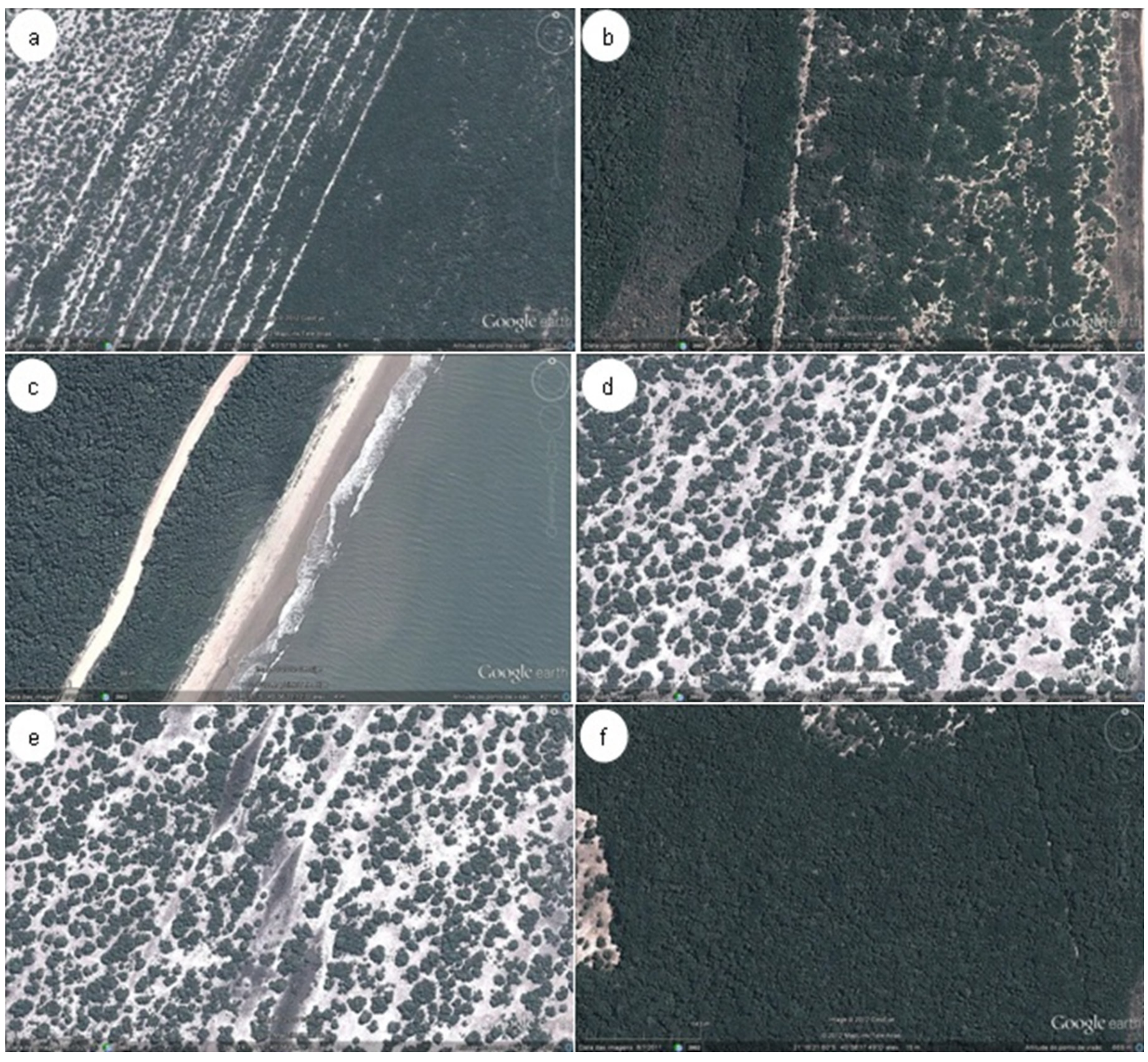

Figura 3. Restinga de Praia das Neves, ES: a) linhas de deposição de sedimentos paralelas a costa. b) trechos das formações Herbácea fechada de cordão arenoso, Herbácea fechada inundada e Floresta de cordão arenoso. c) trechos das formações Halófila-psamófila reptante, Arbustiva fechada de pós-praia e Floresta de cordão arenoso. d) trechos das formações Arbustiva aberta não-inundável, com cordões de vegetação herbácea não inundável. e) trechos da formação Arbustiva aberta não-inundável com cordões de vegetação herbácea inundável. f) Floresta de cordão arenoso (Google Earth, 2012: http://earth.google.com).

Figure 3. Sandy coastal plain (restinga) of "Praia das Neves", ES, Brazil: a) Lines of sediment deposition parallel to the coast. b) Stretches of herbaceous communities of Ridge palmoid and Permanently flooded sedge swamps, and Ridge forest. c) Stretches of herbaceous communities of Beach graminoid, Shrub communities of Beach thicket and Ridge forest. d) Stretches of shrub communities of non-flooded scrub with ridges of not flooded herbaceous vegetation. e) Stretches of shrub communities of non-flooded scrub with ridges of seasonally flooded herbaceous vegetation. f) Ridge forest (Google Earth, 2012: http:// earth.google.com).

Blutaparon portulacoides, Canavalia rosea, Ipomoea pescaprae, I. imperati, Panicum racemosum e Sporobolus virginicus.

Herbácea fechada de cordão arenoso - Em alguns trechos ao longo da costa, especialmente naqueles próximos aos fragmentos florestais remanescentes, ocorre uma mudança abrupta da vegetação da praia para vegetação arbustiva. Em outros trechos a vegetação herbácea de praia vai gradualmente se adensando e havendo um predomínio visível de gramíneas com alguns outros elementos, como Cereus fernambucensis e Allagoptera arenaria, o guriri-dapraia (Figura 4e, 4f). Ainda na vertente externa do cordão arenoso voltada para o oceano, em alguns raros trechos, A. arenaria pode formar conjunto adensado, constituindo uma vegetação fechada. Às vezes, essa espécie predomina em áreas, porém de forma não adensada, entremeada por solo mais ou menos desnudo. A. arenaria é uma espécie registrada em praticamente todas as formações vegetais onde, em geral, ocorre esparsamente no meio de outras espécies. $\mathrm{Na}$ restinga da Marambaia, Menezes \& Araújo (2005) verificaram que A. arenaria domina cristas praianas na vertente externa do cordão arenoso. Os referidos autores consideram esse tipo de vegetação uma formação natural por ser mencionada em bibliografias antigas 

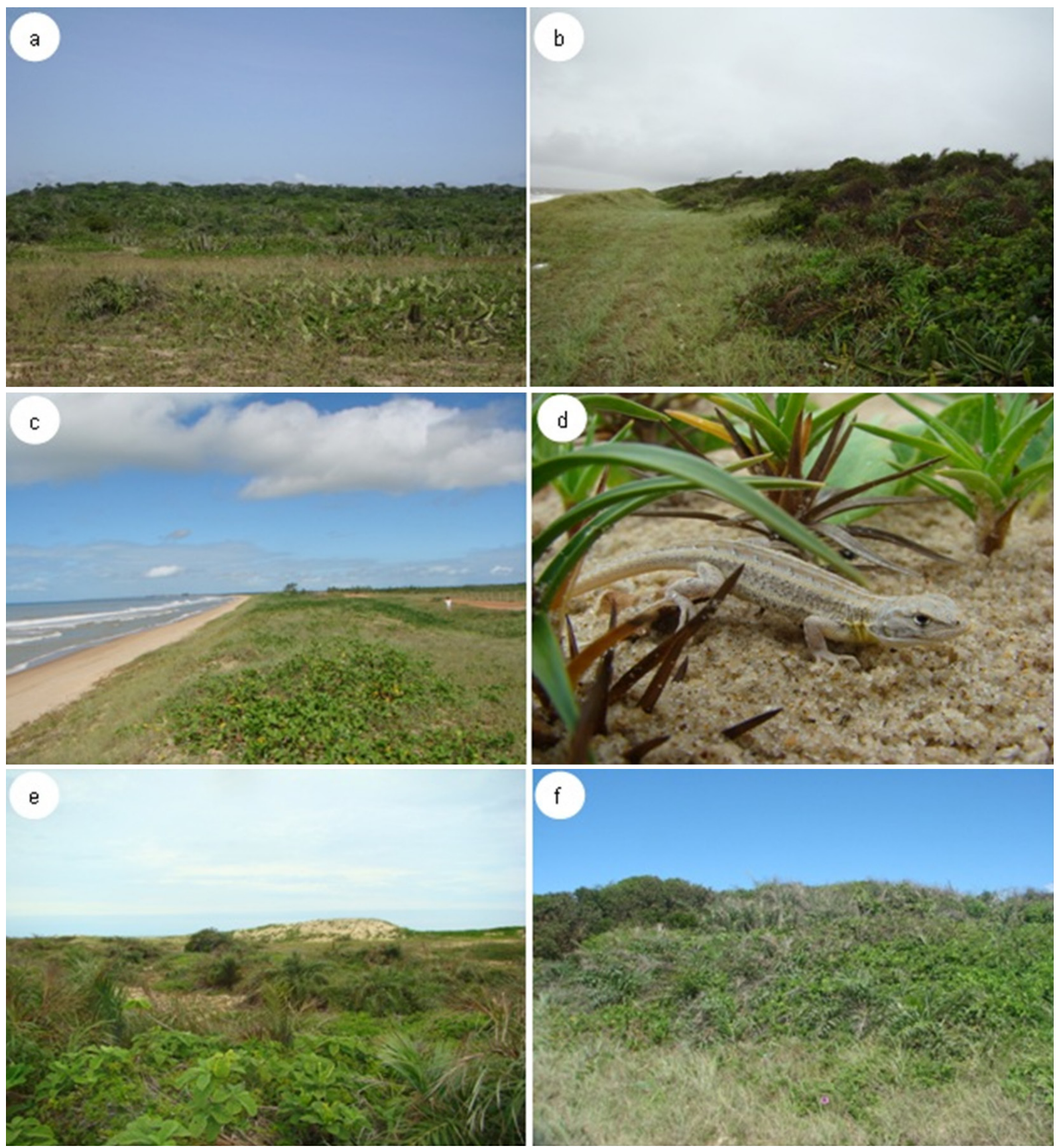

Figura 4. Restinga de Praia das Neves, ES. a) Fitofisionomias herbáceas, arbustivas e florestal em cordões paralelos a costa. b) Fitofisionomias herbácea e arbustiva. c) Faixa da formação Halófila-psamófila repstante. d) Formação Halófila-psamófila reptante e a largatixa-de-areia, Liolaemus lutzae, em seu habita natural. e) Faixa da formação Halófila-psamófila reptante em ecótono com fitofisionomias herbácea fechada e arbustiva fechada. f) Formação Herbácea fechada de cordão arenoso caracterizada pelo adensamento de guriri-da-praia (Allagoptera arenaria).

Figure 4. Sandy coastal plain (restinga) of "Praia das Neves", ES, Brazil. a) Herbaceous, shruby and arboreous phytophysiognomies in ridges paralles to the coast. b) Herbaceous and shruby phytophysiognomies. c) Stretches of Beach graminoid communities. d) Beach graminoid community and the sandy-lizard, Liolaemus lutzae, in its natural habitat. e) Ridge of the Beach graminoid community in ecotone with the Ridge palmoid and Beach thicket communities. f) Ridge palmoid community characterized by the high density of Allagoptera arenaria (guriri-da-praia).

e encontrada em diversas restingas fluminenses e capixabas. Para Pereira (2003) esta formação é ocasionada por alteração antrópica. Entretanto, ainda não existem dados suficientes que comprovem esse fato, visto que tanto na Marambaia, como em Praia das Neves, a vegetação natural foi alterada.

Herbácea fechada inundada - Em depressões em meio aos cordões arenosos, entre a vegetação herbácea pós-praia e a vegetação florestada da restinga, assim como em extensas áreas próximas ao rio Itabapoana, ocorrem áreas brejosas paralelas à linha da costa, dominadas especialmente por Typha domingensis (tabôa), cuja altura pode chegar a 2,5 $\mathrm{m}$ (Figura $5 \mathrm{a}$ ).

Herbácea fechada inundável - Alguns trechos no interior da restinga são ocupados exclusivamente por espécies de pequeno porte, esparsas em solo predominantemente desnudo, formando 
faixas paralelas à costa. Ocasionalmente pode se apresentar em faixas inundáveis, ocorrendo em trechos de maior profundidade e afloramento do lençol freático (Figura $5 \mathrm{~b}, 5 \mathrm{c}$ ), com vegetação herbácea e arbustiva de pequeno porte e com algumas gramíneas. Raramente encraves inundáveis no interior da restinga de Praia das Neves são ocupados por vegetação herbácea de pequeno porte mais densa, dominada por gramíneas (Figura 5c). Menezes \& Araújo (2005) verificaram na restinga da Marambaia sua ocorrência em maiores extensões após a formação halófila-psamófila formando lagoas temporárias, dominadas por representantes de Poaceae e Cyperaceae. Logo após o Rio Itabapoana, na costa do Rio de Janeiro, observa-se a formação de lagoas imediatamente após a mesma formação, equivalente às descrita por Menezes \& Araújo (2005), e que são comuns ao longo de todas as demais restingas fluminenses. Diferentemente, em Praia das Neves, as áreas brejosas ou alagáveis se restringem a pequenos trechos de encrave em outros tipos de vegetação no interior da restinga, o que poderia ser explicado por uma dinâmica costeira de sedimentação e de formação diferenciada de cordões arenosos (Muehe 1984, Pereira et al. 1992).

Ainda considerando a vegetação herbácea, no interior de outras formações predominantes ocorrem cordões mais elevados, não sujeitos a inundações (Figura 5d), onde são encontrados indivíduos herbáceos a arbustivos de pequeno porte e dispostos esparsamente. Dentre esses, Actinocephallus ramosus forma populações de
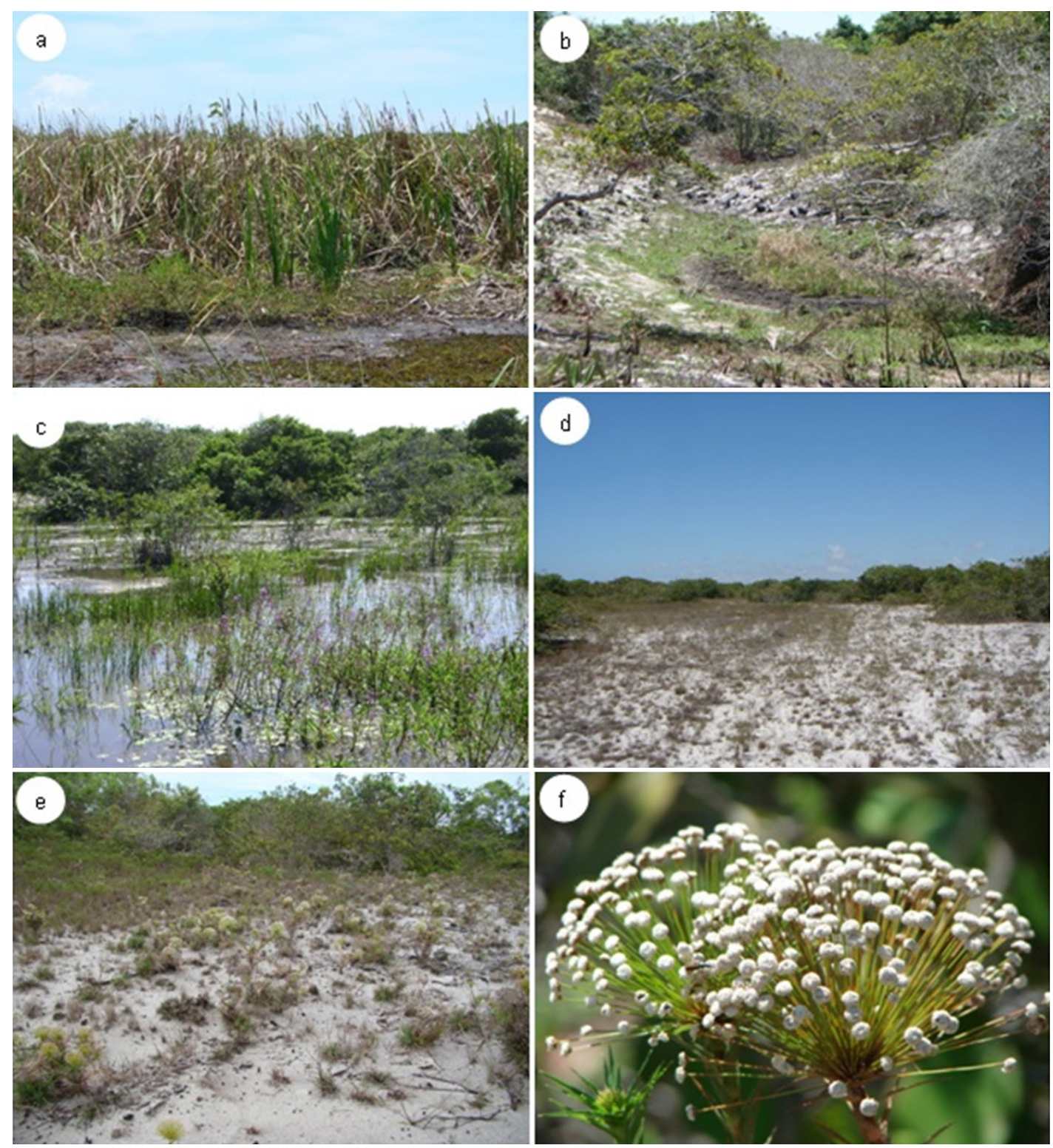

Figura 5. Restinga de Praia das Neves, ES. a) Área brejosa dominada por Typha domingensis (tabôa) caracterizando a formação Herbácea fechada inundada. b) Faixa inundável no interior de fitofisionomia arbustiva aberta. c) Faixa inundável no interior de fitofisionomia arbustiva aberta. d) Faixa de vegetação arbustivoherbácea no interior de fitofisionomia arbustiva aberta. e) Faixa de vegetação arbustivo-herbácea no interior da formação Arbustiva aberta não inundável, com população de Actinocephallus ramosus. f) Inflorescência de Actinocephallus ramosus.

Figure 5. Sandy coastal plain (restinga) of "Praia das Neves", ES, Brazil. a) Swamp with dense population of Typha domingensis (tabôa), wich characterizes the Permanently flooded sedge swamps community. b) Flooded ridge in the interior of the non-flooded scrub phytophysiognomy. c) Flooded ridge in the interior of the non-flooded scrub phytophysiognomy. d) Ridge of shruby-herbaceous vegetation in the interior of the non-flooded scrub phytophysiognomy. e) Ridge of shruby-herbaceous vegetation in the interior of the non-flooded scrub community, with population of Actinocephallus ramosus. f) Inflorescence of Actinocephallus ramosus. 
indivíduos ocupando longas extensões (Figura 5e, 5f) e outras plantas típicas desses cordões na restinga de Praia das Neves são Senna appendiculata, Chamaecrista desvauxii e Marcetia taxifolia. (Figura 6a, 6b). Trechos extensos de vegetação herbácea no domínio da formação arbustiva aberta não inundável, de forma semelhante ao verificado na restinga Praia das Neves, também foram descritos para a restinga de Jurubatiba (Montezuma \& Araújo 2007) e não foram tratados como diferentes fitofisionomias. Assim, a vegetação esparsa de pequenos arbustos e ervas no interior da formação arbustiva aberta não-inundável foi considerada parte da fitofisionomia arbustiva aberta, descrita adiante.

\section{Fitofisionomias arbustivas}

Arbustiva fechada de pós-praia - Após a formação halófilapsamófila ocorre o incremento gradual até uma formação arbustiva fechada, que também se posiciona paralela à costa. Entretanto, em alguns trechos logo após este tipo de formação ocorre uma mudança abrupta na vegetação para o hábito arbustivo, constituindo uma formação densa e dominada por Jacquinia armillaris, às vezes entremeada por grupamentos de Allagoptera arenaria (Figura 6c, 6d). Essa vegetação na área estudada constitui uma faixa estreita e restrita a pequenos fragmentos ao longo da costa (Figura 3c), mas originalmente deveria ocupar maiores extensões da praia. As plantas
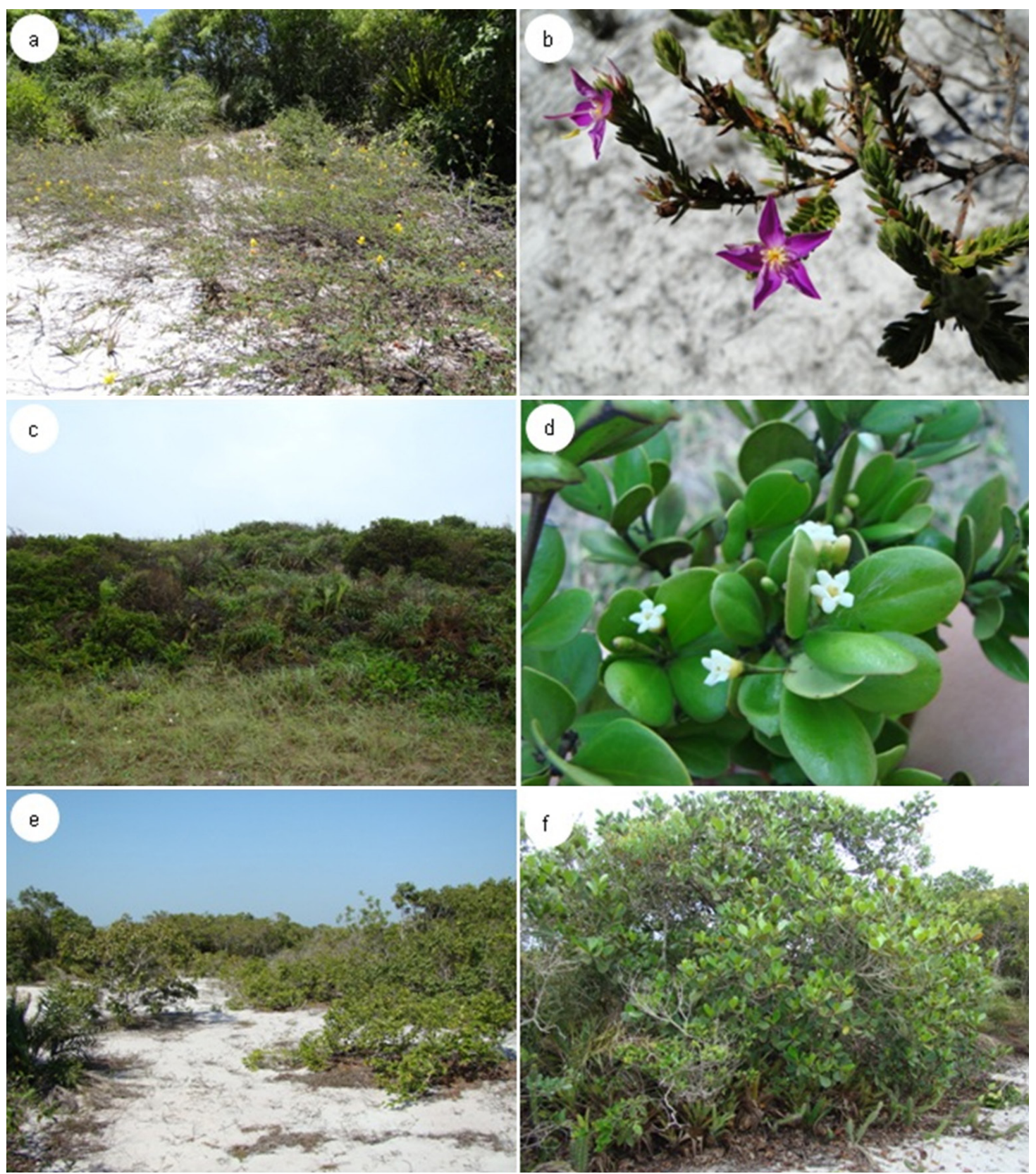

Figura 6. Restinga de Praia das Neves, ES. a) Chamaecrista desvauxii em fitofisionomia arbustiva aberta. b) Marcetia taxifolia em fitofisionomia arbustiva aberta. c) Formação Arbustiva fechada de pós-praia. d) Jacquinia armillaris em fitofisionomia arbustiva fechada. e) Formação Arbustiva aberta não inundável. f) Clusia fluminensis formando moitas na fitofisionomia arbustiva aberta não inundável.

Figure 6. Sandy coastal plain (restinga) of "Praia das Neves", ES, Brazil. a) Chamaecrista desvauxii in the non-flooded scrub phytophysiognomy. b) Marcetia taxifolia in the non-flooded scrub phytophysiognomy. c) Beach Thicket community. d) Jacquinia armillaris in the beach thicket phytophysiognomy. e) Non flooded scrub community. f) Clusia fluminensis forming thickets in the non-flooded scrub phytophysiognomy. 
arbustivas, com aproximadamente $1 \mathrm{~m}$ de altura, vão gradativamente sendo substituídas por espécies arbóreas na direção do interior, até constituírem vegetação florestal. Da mesma forma, como verificado na Praia das Neves, uma transição abrupta entre a formação halófilapsamófila reptante e Arbustiva fechada de pós-praia foi descrita para a restinga da Marambaia (Menezes \& Araújo 2005).

Arbustiva aberta não inundável - A vegetação que ocupa as maiores extensões na restinga de Praia das Neves é composta por moitas densas, com altura de até $6 \mathrm{~m}$, com as moitas esparsas, na forma de ilhas rodeadas por solo praticamente desnudo (Figura 6e). Em geral, ocorre sempre um elemento arbóreo central, comumente Clusia fluminensis, circundada por plantas arbustivas e herbáceas adensadas (Figura 6f). Estas, por sua vez, são rodeadas por ervas esparsas, evidenciando uma diminuição abrupta da altura e densidade dos componentes. Caracterizam-se nessa formação pelo menos dois segmentos ou estandes distintos: as áreas de areia com vegetação herbácea esparsa e as ilhas de vegetação, essas em diferentes estádios de desenvolvimento (Pereira et al. 2004). Está localizada nos cordões arenosos mais internos, após a floresta ou a formação arbustiva póspraia e ocorre mais ou menos alinhada ao longo de faixas (Figura 7a).
Plantas típicas dessa formação são Byrsonima sericea, Cupania emarginata, Ocotea notata, Protium icicariba e Senna appendiculata dentre os elementos arbustivos e arbóreos, além de Clusia fluminensis. Mais externamente são comuns bromélias (Figura 7b), orquídeas e ervas de baixo porte ou reptantes. Essa formação arbustiva aberta é aparentemente restrita ao Rio de Janeiro, Espírito Santo e Sul da Bahia (Menezes \& Araújo 2005). Embora nas restingas de Maricá e de Jurubatiba, assim como na de Praia das Neves, o elemento central comumente seja do gênero Clusia, para outras áreas podem ser observadas outras espécies (Menezes \& Araújo 2005, Magnago 2007, Araújo et al. 2009), corroborando uma classificação mais generalista para a formação. Ainda que no centro das moitas ocorra sempre um elemento arbóreo, a maioria das espécies ocorrentes nessa vegetação é de arbustos. Além disso, as espécies comumente apresentam ramificações desde a base do caule, também adquirindo um aspecto arbustivo.

Ainda não é possível afirmar se as moitas esparsas ocorrem onde uma vegetação primária sofreu alteração, ou se são locais onde os fatores edáficos só permitem aquele tipo de vegetação, ou se por ambos os fatores. Na restinga da Marambaia, assim como na da
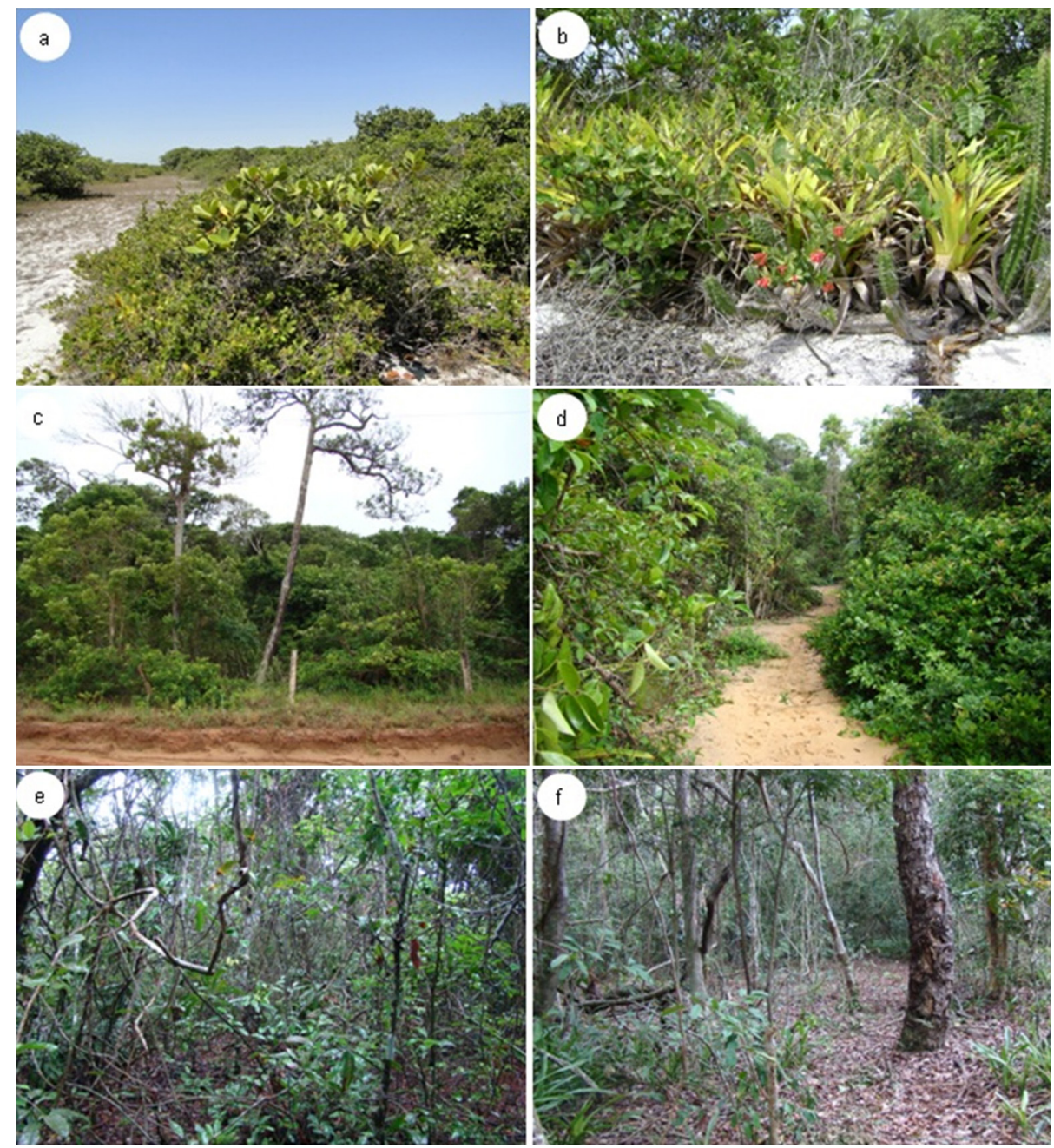

Figura 7. Restinga de Praia das Neves, ES. a) Faixa de vegetação arbustivo-herbácea no interior da formação Arbustiva aberta não inundável. b) Espécies arbustivas e herbáceas no entorno das moitas na fitofisionomia arbustiva aberta não inundável. c) Floresta de cordão arenoso. d) Trilha no interior da floresta. e) Interior da floresta, com lianas e outros elementos do sub-bosque. f) Interior da floresta com sub-bosque herbáceo em alguns trechos.

Figure 7. Sandy coastal plain (restinga) of "Praia das Neves", ES, Brazil. a) Ridge of shruby-herbaceous vegetation in the interior of non-flooded scrub phytophysiognomy . b) Shruby and herbaceous species around the tickets in the non-flooded scrub phytophysiognomy. c) Ridge forest. d) Trail in the interior of the Ridge forest. e) Interior of the forest, showing lianas and other members of the understory. f) Interior of the forest with herbaceous understory in some stretches. 
Praia das Neves, os vestígios de alteração nesse tipo de formação são evidentes. Aparentemente, na restinga estudada algumas áreas devastadas, que anteriormente eram ocupadas por florestas, são atualmente dominadas por vegetação arbustiva aberta (Figura 3f).

\section{Fitofisionomia arbórea}

Floresta de cordão arenoso - As áreas florestadas da restinga de Praia das Neves se restringem atualmente a fragmentos, às vezes adjacentes a pequenas manchas de áreas alagadas (Formação Herbácea Fechada Inundada). As plantas arbóreas dominam esta fisionomia (Figura 7c, 7d) e o dossel atinge de 15 a 20 m de altura, com algumas árvores emergentes. $\mathrm{O}$ sub-bosque é rico em lianas, arbustos, ervas e epífitas, às vezes com esparsa cobertura vegetal (Figura 7e, 7f). A serapilheira é densa e contínua e os elementos arbóreos de maior porte são escassos. Destacam-se Aechmea floribunda,
Anchietea pyrifolia e Schaueria lophura além das arbóreas Allophylus puberulus, Andira nitida, Brasiliopuntia brasiliensis, Byrsonima sericea, Clusia fluminensis, Machaerium lanceolatum, Ocotea notata, Stephanopodium sessile, Tapirira guianensis e Zollernia glabra. Magnago et al. (2011) destacam essa como a formação de maior riqueza nas restingas do Rio de Janeiro e do Espírito Santo, como também foi verificado nesse estudo. Isso pode se dar não só pela ocorrência de epífitas, conforme salientam Magnago et al. (2011), mas sim pela presença das diversas formas de vida, que ocupam os diferentes estratos da floresta. Na restinga estudada a formação florestal está localizada logo após a formação Arbustiva Fechada de Pós-Praia. Observando-se a Figura 8, retirada de Dias \& Silva (1984), nota-se que a área de floresta era mais extensa no passado, formando uma faixa mais contínua.

Outras espécies verificadas nas diferentes formações na restinga de Praia das Neves estão listadas na Tabela 1.

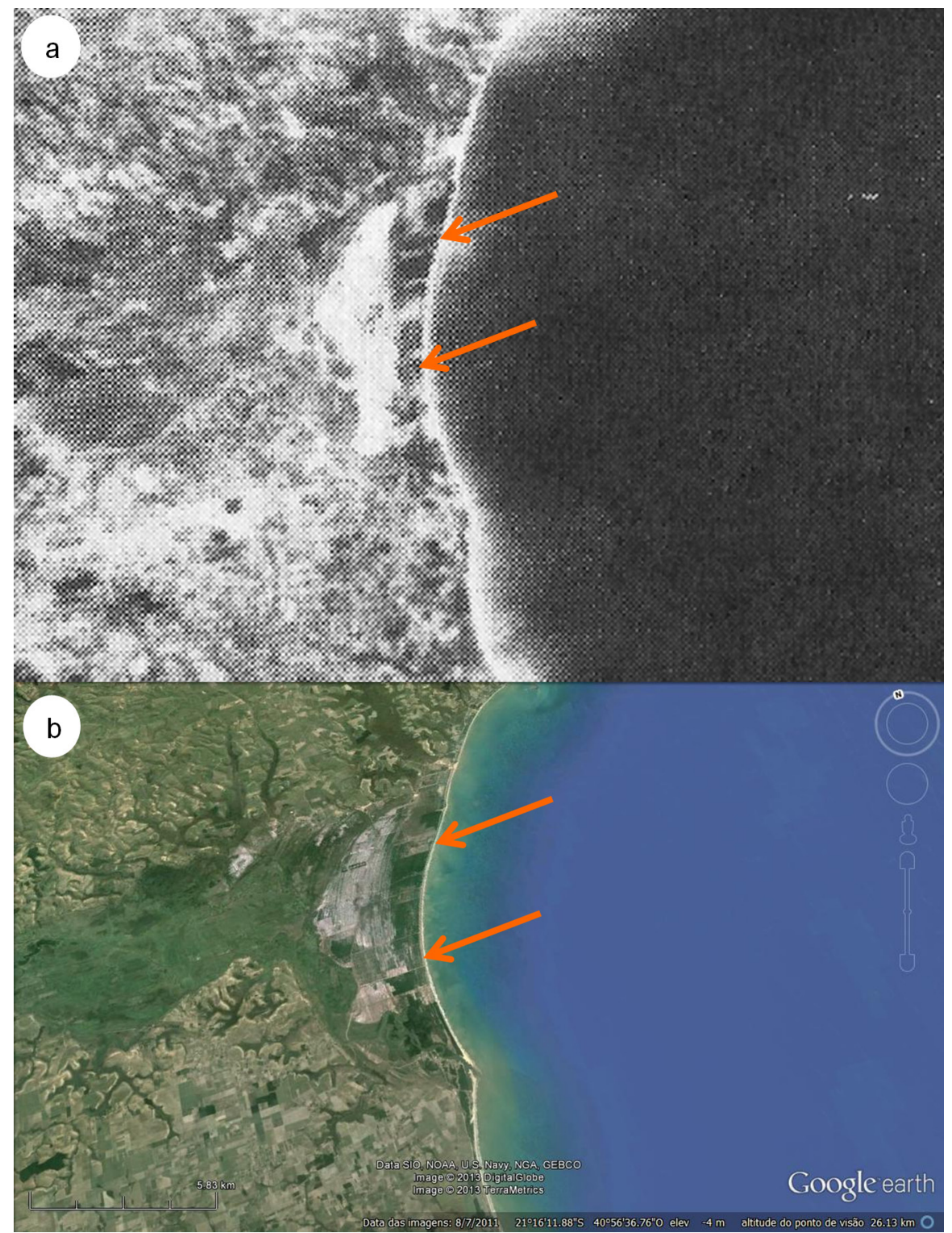

Figura 8. a) Imagem do Satélite Landsat (retirada de Dias \& Silva 1984) ampliada, evidenciando a restinga de Praia das Neves, ES. b) Imagem recente da restinga em escala aproximada (Google Earth 2012: http://www.earth.google.com), com a redução na cobertura vegetal, ao longo dos anos, indicada por círculos.

Figure 8. a) Image of the Landsat satelite (source: Dias \& Silva 1984) amplified, showing the sandy coastal plain (restinga) of "Praia das Neves", ES, Brazil. b) Current image of the restinga in approximate scale (Google Earth 2012: http://www.earth.google.com), showing the reduction of the vegetal cover over the years, indicated by circles. 
Tabela 1. Espécies vasculares coletadas na Restinga de Praia das Neves, ES, acompanhadas de seu registro no Herbário RBR, hábito, formação vegetal e distribuição nos principais biomas brasileiros. (Hábito: ab: arbusto; av: árvore; er: erva; ep: epífita; li: liana. tr: trepadeira herbácea. Formações vegetais: Aa: Arbustiva aberta não inundável; Af: Arbustiva fechada de pós-praia; Fl: Floresta de cordão arenoso; Hf: Herbácea fechada de cordão arenoso; Hi: Herbácea fechada inundada; Hv: Herbácea fechada inundável; Hp: Halófila-psamófila reptante. Mg: manguezal. Distribuição: AM: Amazônia; CA: Caatinga; CE: Cerrado; MA: Floresta Atlântica; PA: Pampa; PN: Pantanal; EX: exótica).

Table 1. Vascular species collected at the sandy coastal plain (restinga) of "Praia das Neves", ES, Brazil, followed by its register number at the RBR Herbarium, habit, plant community and distribuition in the main Brazilian biomes. (Habit: ab: shrub; av: tree; er: herb; ep: epyphit; li: liana. tr: herbaceous climber. Plant communities: Non flooded scrub; Af: Beach thicket; Fl: Ridge forest; Hf: Ridge palmoid; Hi: Permanently flooded sedge swamps; Hv: Seasonally flooded sedge swamps; Hp: Beach graminoid. Mg: mangrove. Distribuition: AM: Amazonia; CA: Caatinga; CE: Cerrado; MA: Atlantic Forest; PA: Pampa; PN: Pantanal; EX: exotic).

\begin{tabular}{|c|c|c|c|}
\hline $\begin{array}{c}\text { FAMÍLIA } \\
\text { Espécie (material testemunho, RBR) }\end{array}$ & Hábito & $\begin{array}{c}\text { Formação } \\
\text { vegetal }\end{array}$ & Distribuição \\
\hline \multicolumn{4}{|l|}{ ACANTHACEAE } \\
\hline Avicennia schaueriana L. [D.M.Braz et al. 419] & av & $\mathrm{Mg}$ & AM, MA \\
\hline Justicia cydoniifolia (Nees) Lindau [D.M.Braz et al. 219] & $\mathrm{ab}$ & Fl & MA \\
\hline Ruelia solitaria Vell. [M.M.T.Rosa et al. 532] & er & $\mathrm{Fl}$ & MA \\
\hline Schaueria lophura Nees [D.M.Braz et al. 197] & $a b$ & $\mathrm{~F} 1$ & MA \\
\hline Thunbergia alata Poir. [D.M.Braz et al. 422] & li & $\mathrm{Fl}$ & EX \\
\hline \multicolumn{4}{|l|}{ AMARANTHACEAE } \\
\hline Blutaparon portulacoides (A. St.-Hil.) Mears [G.V.Somner et al. 1196] & er & Hр & $\mathrm{CE}, \mathrm{MA}$ \\
\hline Gomphrena vaga Mart. [D.M.Braz et al. 202] & er & $\mathrm{F} 1$ & $\mathrm{AM}, \mathrm{CA}, \mathrm{CE}, \mathrm{MA}$ \\
\hline Hebanthe eriantha (Poir.) Pedersen [D.M.Braz et al. 187] & er & $\mathrm{F} 1$ & $\mathrm{AM}, \mathrm{CE}, \mathrm{MA}$ \\
\hline \multicolumn{4}{|l|}{ ANACARDIACEAE } \\
\hline Schinus terebinthifolius Raddi [G.V.Somner et al. 1200] & av & Af & CA, MA, PA \\
\hline Tapirira guianensis Aubl. [M.M.T.Rosa et al. 508] & av & F1 & $\mathrm{AM}, \mathrm{CA}, \mathrm{CE}, \mathrm{MA}, \mathrm{PA}, \mathrm{PN}$ \\
\hline \multicolumn{4}{|l|}{ APOCYNACEAE } \\
\hline Forsteronia pilosa Müll. Arg. [G.V.Somner et al. 1222] & li & $\mathrm{F} 1$ & MA \\
\hline Mandevilla guanabarica Casar. ex M.F.Sales [E.L.Jacques et al. 1688] & li & $\mathrm{Aa}$ & MA \\
\hline Oxypetalum banksii R.Br. ex Schult. [D.M.Braz et al. 182] & $\operatorname{tr}$ & $\mathrm{Aa} ; \mathrm{Fl}$ & CE, MA \\
\hline Matelea maritima (Vell.) Fontella [A.V.S.Couto et al. s/n] & $\operatorname{tr}$ & $\mathrm{Aa}$ & AM, MA \\
\hline Peplonia asteria (Vell.) Fontella \& E.A. Schwarz. [E.L.Jacques et al. 1701] & $\operatorname{tr}$ & $\mathrm{Aa}$ & MA \\
\hline \multicolumn{4}{|l|}{ ARACEAE } \\
\hline Anthurium intermedium Kunth [M.M.T.Rosa et al. 533] & er & Fl & MA \\
\hline Anthurium minarum Sakur. \& Mayo [D.M.Braz et al. 212] & er & $\mathrm{F} 1$ & $\mathrm{CE}, \mathrm{MA}$ \\
\hline Anthurium aff. parasiticum (Vell.) Stellfeld. [E.L.Jacques et al. 1708] & er & Aa & MA \\
\hline \multicolumn{4}{|l|}{ ARECACEAE } \\
\hline Allagoptera arenaria (Gomes) Kuntze [E.L.Jacques et al. 1683] & er & Aa; Af; Hf; Hp & MA \\
\hline \multicolumn{4}{|l|}{ ASTERACEAE } \\
\hline Baccharis trinervis (Lam.) Pers. [D. M.Braz et al. 195] & $\operatorname{tr}$ & $\mathrm{F} 1$ & $\mathrm{AM}, \mathrm{CA}, \mathrm{CE}, \mathrm{MA}$ \\
\hline Emilia forsbergii Nicolson [Observação local] & er & Hр & $\mathrm{AM}, \mathrm{CA}, \mathrm{CE}, \mathrm{MA}, \mathrm{PA}, \mathrm{PN}$ \\
\hline Emilia sonchifolia DC. [G.V.Somner et al. 1192] & er & Нp & $\mathrm{AM}, \mathrm{CA}, \mathrm{CE}, \mathrm{MA}$ \\
\hline Porophyllum ruderale (Jacq.) Cass. [G.V.Somner et al. 1197] & er & Hр & MA \\
\hline Tridax procumbens L. [G.V.Somner et al. 1194] & er & $\mathrm{Hp}$ & $\mathrm{AM}, \mathrm{CA}, \mathrm{CE}, \mathrm{MA}, \mathrm{PA}, \mathrm{PN}$ \\
\hline Trixis antimenorrhoea (Schrank) Kuntze [G.V.Somner et al. 1209] & er & Hf & CE, MA \\
\hline \multicolumn{4}{|l|}{ BIGNONIACEAE } \\
\hline Anemopaegma cf. citrinum Mart. ex DC. [Observação local] & li & Aa & CE, MA \\
\hline Fridericia conjugata (Vell.) L.G.Lohmann [G.V. Somner et al. 1211] & li & $\mathrm{F} 1$ & AM, CE, MA, PA \\
\hline Jacaranda puberula Cham. [M.M.T. Rosa et al. 503] & av & $\mathrm{F} 1$ & MA \\
\hline Lundia cordata (Vell.) DC. [M.M.T. Rosa et al. 528] & li & $\mathrm{F} 1$ & $\mathrm{CA}, \mathrm{MA}$ \\
\hline \multicolumn{4}{|l|}{ BORAGINACEAE } \\
\hline Varronia curassavica Jacq. [G.V. Somner et al. 1208] & av & $\mathrm{Hf}$ & $\mathrm{AM}, \mathrm{CA}, \mathrm{MA}$ \\
\hline \multicolumn{4}{|l|}{ BROMELIACEAE } \\
\hline Aechmea blanchetiana (Baker) L.B.Sm.[ Observação local] & er & Aa & MA \\
\hline Aechmea floribunda Mart. ex Schult. f. [D. M.Braz et al. 204] & er & F1 & MA \\
\hline Aechmea nudicaulis Griseb. [G.V.Somner et al. 1266] & er & $\mathrm{Aa}$ & MA \\
\hline Aechmea patentissima Baker [D. M.Braz et al. 181] & er & $\mathrm{Aa}$ & MA \\
\hline Aechmea saxicola L.B.Sm. [Observação local] & er & $\mathrm{Aa}$ & MA \\
\hline
\end{tabular}


Tabela 1. Continuação....

Table 1. Continued...

\begin{tabular}{|c|c|c|c|}
\hline $\begin{array}{c}\text { FAMÍLIA } \\
\text { Espécie (material testemunho, RBR) }\end{array}$ & Hábito & $\begin{array}{c}\text { Formação } \\
\text { vegetal }\end{array}$ & Distribuição \\
\hline Billbergia amoena (Lodd.) Lindl. [Observação local] & er & $\mathrm{Fl}$ & $\mathrm{AM}, \mathrm{CE}$ \\
\hline Neoregelia cruenta (Graham) L.B.Sm. [G.V.Somner et al. 1225] & er & $\mathrm{Aa}$ & MA \\
\hline Tillandsia stricta Sims [M.M.T.Rosa et al. 540] & ep & $\mathrm{Aa} ; \mathrm{Fl}$ & $\mathrm{CE}, \mathrm{MA}$ \\
\hline \multicolumn{4}{|l|}{ BURSERACEAE } \\
\hline Protium heptaphyllum March. [D.M.Braz et al. 218] & av & $\mathrm{Aa} ; \mathrm{Fl}$ & AM, MA \\
\hline Protium icicariba March. [E.L.Jacques et al. 1704] & av & Aa & MA \\
\hline \multicolumn{4}{|l|}{ CACTACEAE } \\
\hline Brasiliopuntia brasiliensis (Willd.) A. Beger [G.V.Somner et al. 1443] & av & $\mathrm{Fl}$ & $\mathrm{CA}, \mathrm{CE}, \mathrm{MA}$ \\
\hline Cereus fernambucensis Lem. [M.M.T.Rosa et al. 534] & $\mathrm{ab}$ & Aa; Af; Hp & MA \\
\hline Pereskia aculeata Mill. [G.V.Somner et al. 1215] & av & $\mathrm{Fl}$ & CA, CE, MA \\
\hline \multicolumn{4}{|l|}{ CALOPHYLLACEAE } \\
\hline Kielmeyera rizziana Saadi [D.M.Braz et al. 189] & av & F1 & MA \\
\hline \multicolumn{4}{|l|}{ CANNABACEAE } \\
\hline Celtis iguanaea (Jacq.) Sarg. [G.V.Somner et al. 1431] & $a b$ & $\mathrm{Fl}$ & $\mathrm{AM}, \mathrm{CA}, \mathrm{CE}, \mathrm{MA}, \mathrm{PA}, \mathrm{PN}$ \\
\hline \multicolumn{4}{|l|}{ CAPPARACEAE } \\
\hline Capparis flexuosa (L.) L. [G.V.Somner et al. 1214] & $\mathrm{ab}$ & $\mathrm{Fl}$ & MA \\
\hline \multicolumn{4}{|l|}{ CELASTRACEAE } \\
\hline Maytenus obtusifolia Mart. [D.M.Braz et al. 239] & $\mathrm{ab}$ & Af & $\mathrm{CA}, \mathrm{CE}, \mathrm{MA}$ \\
\hline \multicolumn{4}{|l|}{ CHRYSOBALANACEAE } \\
\hline Chrysobalanus icaco L. [A.V.S.Couto et al. s/n] & $a b$ & $\mathrm{Hp}$ & AM, MA \\
\hline Licania hoehnei Pilg. [M.M.T.Rosa et al. 526] & av & $\mathrm{Fl}$ & $\mathrm{CE}, \mathrm{MA}$ \\
\hline \multicolumn{4}{|l|}{ CLUSIACEAE } \\
\hline Clusia fluminensis Planch. \& Triana [M.M.T.Rosa et al .519] & av & $\mathrm{Fl}$ & MA \\
\hline \multicolumn{4}{|l|}{ COMMELINACEAE } \\
\hline Commelina erecta L. [M.V.L. Pereira-Moura 960] & er & $\mathrm{Hp}$ & $\mathrm{AM}, \mathrm{CA}, \mathrm{CE}, \mathrm{MA}$ \\
\hline \multicolumn{4}{|l|}{ CONVOLVULACEAE } \\
\hline Ipomoea imperati (Vahl) Griseb. [D.M.Braz et al. 242] & er & $\mathrm{Hp}$ & AM, MA \\
\hline Ipomoea pes-caprae (L.) R.Br. [G.V. Somner et al. 1190] & er & $\mathrm{Hp}$ & AM, MA \\
\hline \multicolumn{4}{|l|}{ CYPERACEAE } \\
\hline Bulbostylis capillaris C.B.Clarke [M.M.T. Rosa et al. 524] & er & F1 & CA, CE, MA, PA \\
\hline Cyperus ligularis L. [D.M.Braz et al. 255] & er & Aa & $\mathrm{AM}, \mathrm{CA}, \mathrm{CE}, \mathrm{MA}$ \\
\hline Fimbristylis autumnalis Roem. \& Schult. [E.L.Jacques et al. 1715] & er & Aa & $\mathrm{AM}, \mathrm{CE}, \mathrm{AM}$ \\
\hline Remirea maritma Aubl [A.V.S. Couto et al. s/n] & er & $\mathrm{Hp}$ & MA \\
\hline \multicolumn{4}{|l|}{ DICHAPETALACEAE } \\
\hline Stephanopodium sessile Rizzini [D.M.Braz et al. 214] & av & F1 & MA \\
\hline \multicolumn{4}{|l|}{ ERICACEAE } \\
\hline Gaylussacia brasiliensis (Spreng.) Meisn. [E.L.Jacques et al.1690] & $\mathrm{ab}$ & Aa & CA, CE, MA \\
\hline \multicolumn{4}{|l|}{ ERIOCAULACEAE } \\
\hline Actinocephalus ramosus (Wikstr.) Sano [D.M.Braz et al. 421] & er & Aa & $\mathrm{CA}, \mathrm{CE}, \mathrm{MA}$ \\
\hline \multicolumn{4}{|l|}{ ERYTHROXYLACEAE } \\
\hline Erythroxylum ovalifolium Peyr. [M.M.T.Rosa et al. 521] & $a b$ & $\mathrm{Aa} ; \mathrm{Fl}$ & MA \\
\hline \multicolumn{4}{|l|}{ EUPHORBIACEAE } \\
\hline Dalechampia micromeria Baill. [G.V.Somner et al. 1228] & $\operatorname{tr}$ & $\mathrm{Fl}$ & MA \\
\hline Dalechampia pentaphylla Lam. [G.V.Somner et al. 1437] & li & $\mathrm{Fl}$ & MA \\
\hline Euphorbia hyssopifolia L. [D.M.Braz et al. 244 & er & $\mathrm{Hp}$ & $\mathrm{AM}, \mathrm{CA}, \mathrm{CE}, \mathrm{MA}, \mathrm{PA}$ \\
\hline Microstachys corniculata (Vahl) Griseb. [M.M.T.Rosa et al. 509] & er & $\mathrm{Fl}$ & MA \\
\hline \multicolumn{4}{|l|}{ FABACEAE } \\
\hline Andira nitida Mart. ex Benth. [M.M.T.Rosa et al. 504] & av & Fl & MA \\
\hline Canavalia rosea (Sw.) DC. [G.V.Somner et al. 1198] & er & $\mathrm{Hp}$ & AM, MA \\
\hline Centrosema virginianum (L.) Benth. [G.V.Somner et al. 1191] & er & $\mathrm{Hp}$ & $\mathrm{AM}, \mathrm{CA}, \mathrm{CE}, \mathrm{MA}$ \\
\hline Chamaecrista desvauxii (Collad.) Killip [M.M.T.Rosa et al. 512] & er & $\mathrm{Aa} ; \mathrm{Fl}$ & $\mathrm{CE}$ \\
\hline Dalbergia ecastaphyllum (L.) Taub. [D.M.Braz et al. 222] & li & $\mathrm{Mg}$ & AM, MA \\
\hline
\end{tabular}


Tabela 1. Continuação....

Table 1. Continued...

\begin{tabular}{|c|c|c|c|}
\hline $\begin{array}{c}\text { FAMÍLIA } \\
\text { Espécie (material testemunho, RBR) }\end{array}$ & Hábito & $\begin{array}{c}\text { Formação } \\
\text { vegetal }\end{array}$ & Distribuição \\
\hline Indigofera suffruticosa Mill. [G.V.Somner et al. 1205] & $\mathrm{ab}$ & $\mathrm{Aa}$ & $\mathrm{AM}, \mathrm{CA}, \mathrm{CE}, \mathrm{MA}$ \\
\hline Inga vera Willd [A.F.Nunes 08 ] & av & Fl & $\mathrm{AM}, \mathrm{CA}, \mathrm{CE}, \mathrm{MA}$ \\
\hline Machaerium lanceolatum (Vell.) J.F.Macbr. [D.M.Braz et al. 188] & av & $\mathrm{Fl}$ & $\mathrm{AM}, \mathrm{CE}, \mathrm{MA}$ \\
\hline Macroptilium lathyroides (L.) Urb. [D.M.Braz et al. 236] & $\operatorname{tr}$ & Af & $\mathrm{AM}, \mathrm{CA}, \mathrm{CE}, \mathrm{MA}$ \\
\hline Ormosia arborea (Vell.) Harms [Observação local] & ar & $\mathrm{Mg}$ & CE, MA \\
\hline Senegalia lowei (L.Rico) Seigler \& Ebinger [G .V.Somner et al. 1430] & $\mathrm{ab}$ & Af & MA \\
\hline Senna appendiculata (Vogel) Wiersema [G.V.Somner et al. 1227] & ar & Aa & MA \\
\hline Sophora tomentosa L. [D.M.Braz et al. 246] & ar & Ap & AM, MA \\
\hline Stylosanthes guianensis (Aubl.) Sw. [E.L.Jacques et al. 1684] & er & $\mathrm{Aa} ; \mathrm{Fl} ; \mathrm{Hf}$ & $\mathrm{CE}, \mathrm{MA}$ \\
\hline Vigna caracalla (L.) Verdc. [G.V. Somner et al. 1219] & $\operatorname{tr}$ & $\mathrm{F} 1$ & $\mathrm{AM}, \mathrm{CA}, \mathrm{CE}, \mathrm{MA}$ \\
\hline Zollernia glabra (Spreng.) Yakovlev [D.M.Braz et al. 186] & $a b$ & F1 & MA \\
\hline \multicolumn{4}{|l|}{ LAURACEAE } \\
\hline Cassytha filiformis L. [G.V.Somner et al. 1201] & ep & $\mathrm{Hf}$ & $\mathrm{AM}, \mathrm{CA}, \mathrm{CE}, \mathrm{MA}$ \\
\hline Ocotea notata (Nees \& Mart.) Mez [M.M.T.Rosa et al. 520] & av & $\mathrm{Aa} ; \mathrm{Fl}$ & MA \\
\hline \multicolumn{4}{|l|}{ LYTHRACEAE } \\
\hline Cuphea flava Spreng. [E.L.Jacques et al. 1697] & er & Aa & MA \\
\hline \multicolumn{4}{|l|}{ MALPIGHIACEAE } \\
\hline Byrsonima sericea DC. [E.L.Jacques et al. 1702] & av & $\mathrm{Aa} ; \mathrm{Fl}$ & $\mathrm{CA}, \mathrm{CE}, \mathrm{MA}$ \\
\hline Heteropterys coleoptera A.Juss. [D.M.Braz et al. 184] & li & F1 & MA \\
\hline Peixotoa hispidula A.Juss. [D.M.Braz et al. 199] & $\operatorname{tr}$ & Fl & MA \\
\hline Stigmaphyllon cf. paralias A. Juss. [Observação local] & $\mathrm{ab}$ & Fl & $\mathrm{CA}, \mathrm{CE}, \mathrm{MA}$ \\
\hline \multicolumn{4}{|l|}{ MALVACEAE } \\
\hline Pavonia cancellata (L.) Cav. [D.M.Braz et al. 253] & er & $\mathrm{Aa}$ & $\mathrm{AM}, \mathrm{CA}, \mathrm{CE}, \mathrm{MA}$ \\
\hline Pseudobombax grandiflorum (Cav.) A.Robyns [D.M.Braz et al. 183] & av & Fl & CE, MA \\
\hline Sida planicaulis Cav. [G.V.Somner et al. 1206] & er & Hf & CA, CE, MA, PA \\
\hline \multicolumn{4}{|l|}{ MELASTOMATACEAE } \\
\hline Marcetia taxifolia (St. Hil.) DC. [M.M.T.Rosa et al. 527] & $a b$ & F1 & $\mathrm{AM}, \mathrm{CA}, \mathrm{CE}, \mathrm{MA}$ \\
\hline Pterolepis glomerata (Rottb.) Miq. [E.L.Jacques et al. 1714] & er & Aa & $\mathrm{AM}, \mathrm{CA}, \mathrm{CE}, \mathrm{MA}$ \\
\hline Tibouchina gaudichaudiana Baill. [M.M.T.Rosa et al. 514] & $a b$ & Fl & MA \\
\hline \multicolumn{4}{|l|}{ MOLLUGINACEAE } \\
\hline Mollugo verticillata L. [M.M.T.Rosa et al. 517] & er & Fl; Hp & $\mathrm{AM}, \mathrm{CA}, \mathrm{CE}, \mathrm{MA}, \mathrm{PA}$ \\
\hline \multicolumn{4}{|l|}{ MYRTACEAE } \\
\hline Calyptranthes brasiliensis Spreng. [M.M.T.Rosa et al. 536] & av & F1 & CE, MA \\
\hline Eugenia pisiformis Cambess [A.F.Nunes 06] & av & F1 & MA \\
\hline Eugenia punicifolia (Kunth) DC. [D.M.Braz et al. 194] & av & Fl & $\mathrm{AM}, \mathrm{CA}, \mathrm{CE}, \mathrm{MA}$ \\
\hline Eugenia uniflora L. [A.F.Nunes 07] & av & Fl & CE, MA \\
\hline Neomitranthes obscura (DC.) N.Silveira [E.L.Jacques et al. 1707] & av & Aa & MA \\
\hline \multicolumn{4}{|l|}{ NYCTAGINACEAE } \\
\hline Guapira pernambucensis (Casar.) Lundell [G.V.Somner et al. 1199] & av & $\mathrm{Aa}, \mathrm{Fl}, \mathrm{Hf}$ & MA \\
\hline Pisonia aculeata L. [G.V.Somner et al. 1217] & li & Fl & AM, MA \\
\hline \multicolumn{4}{|l|}{ OCHNACEAE } \\
\hline Ouratea cuspidata (A.St.-Hil.) Engl. [D.M.Braz et al. 220] & $\mathrm{ab}$ & Aa & CE, MA \\
\hline \multicolumn{4}{|l|}{ ORCHIDACEAE } \\
\hline Epidendron denticulatum Barb.Rodr. [E.L.Jacques et al. 1695] & ep & $\mathrm{Aa}$ & $\mathrm{CA}, \mathrm{CE}, \mathrm{MA}$ \\
\hline Oeceoclades maculata (Lindl.) Lindl. [Observação local] & er & Fl & $\mathrm{AM}, \mathrm{CA}, \mathrm{CE}, \mathrm{MA}$ \\
\hline Vanilla sp. [Observação local] & ep & Hf & \\
\hline \multicolumn{4}{|l|}{ PASSIFLORACEAE } \\
\hline Passiflora edulis Sims [G .V.Somner et al. 1435] & li & F1 & $\mathrm{AM}, \mathrm{CA}, \mathrm{CE}, \mathrm{MA}, \mathrm{PA}, \mathrm{PN}$ \\
\hline Passiflora misera Kunth [G.V.Somner et al. 1438] & li & Fl & $\mathrm{AM}, \mathrm{CA}, \mathrm{CE}, \mathrm{MA}$ \\
\hline Passiflora mucronata Lam. [M.M.T. Rosa et al. 510] & li & $\mathrm{Aa} ; \mathrm{Fl}$ & $\mathrm{CA}, \mathrm{CE}, \mathrm{MA}$ \\
\hline \multicolumn{4}{|l|}{ PERACEAE } \\
\hline Pera glabrata (Schott) Baill. [E.L.Jacques et al. 1710] & av & $\mathrm{Aa} ; \mathrm{Fl}$ & $\mathrm{AM}, \mathrm{CA}, \mathrm{CE}, \mathrm{MA}$ \\
\hline
\end{tabular}


Tabela 1. Continuação....

Table 1. Continued...

\begin{tabular}{|c|c|c|c|}
\hline $\begin{array}{c}\text { FAMÍLIA } \\
\text { Espécie (material testemunho, RBR) }\end{array}$ & Hábito & $\begin{array}{l}\text { Formação } \\
\text { vegetal }\end{array}$ & Distribuição \\
\hline \multicolumn{4}{|l|}{ PICRAMNIACEAE } \\
\hline Picramnia glazioviana Engl. [D.M.Braz et al. 215] & av & Fl & MA \\
\hline \multicolumn{4}{|l|}{ PLUMBAGINACEAE } \\
\hline Plumbago scandens L. [G.V.Somner et al. 1204] & $\operatorname{tr}$ & Hf & $\mathrm{AM}, \mathrm{CA}, \mathrm{MA}$ \\
\hline \multicolumn{4}{|l|}{ PHYTOLACCACEAE } \\
\hline Rivina humilis L. [G V.Somner et al. 1432] & er & F1 & AM, CA, MA, PA, \\
\hline \multicolumn{4}{|l|}{ POACEAE } \\
\hline Cenchrus echinatus L. [G.V. Somner et al. 1203] & er & Hf & $\mathrm{AM}, \mathrm{CA}, \mathrm{CE}, \mathrm{MA}, \mathrm{PN}$ \\
\hline Chloris elata Desv. [A.V.S.Couto et al. s/n] & er & $\mathrm{Hp}$ & $\mathrm{CA}, \mathrm{MA}, \mathrm{PA}$ \\
\hline Dactyloctenium aegyptium (L.) Willd. [A.V.S.Couto et al. s/n] & er & Hp & $\mathrm{AM}, \mathrm{CA}, \mathrm{MA}$ \\
\hline Eragrostis ciliaris (L.) R. Br. [G.V.Somner et al. 1202] & er & Hf & $\mathrm{AM}, \mathrm{CA}, \mathrm{CE}, \mathrm{MA}, \mathrm{PN}$ \\
\hline Eragrostis sp. [M.M.T.Rosa et al. 523] & er & Af; Fl & \\
\hline Gymnopogon foliosus (Willd.) Nees [M.M.T.Rosa et al. 538] & er & $\mathrm{F} 1$ & $\mathrm{AM}, \mathrm{CA}, \mathrm{CE}, \mathrm{MA}$ \\
\hline Panicum racemosum (P. Beauv.) Spreng. [A.V.S.Couto et al. s/n] & er & Нp & CA, MA, PA \\
\hline Paspalum arenarium Schrad. [M.M.T.Rosa et al. 525] & er & $\mathrm{F} 1$ & $\mathrm{AM}, \mathrm{CA}, \mathrm{CE}, \mathrm{MA}$ \\
\hline Paspalum maritimum Trin. [D.M.Braz et al. 251] & er & Af & $\mathrm{AM}, \mathrm{CA}, \mathrm{CE}, \mathrm{MA}$ \\
\hline Setaria setosa (Sw.) P. Beauv. [G.V. omner et al. 1436] & er & $\mathrm{Fl}$ & $\mathrm{CA}, \mathrm{MA}$ \\
\hline Sporobolus virginicus Kunth [A.V.S.Couto et al. s/n] & er & Нp & $\mathrm{AM}, \mathrm{CA}, \mathrm{CE}, \mathrm{PA}$ \\
\hline Tricanthecium sp. [E.L.Jacques et al. 1717] & er & $\mathrm{Aa}$ & \\
\hline \multicolumn{4}{|l|}{ POLYGALACEAE } \\
\hline Polygala cyparissias A. St.-Hil. \& Moq. [M.M.T.Rosa et al. 502] & er & Aa; F1 & $\mathrm{CA}, \mathrm{MA}$ \\
\hline \multicolumn{4}{|l|}{ POLYPODIACEAE } \\
\hline Microgramma geminata (Schrad.) R.Tryon \& A.Tryon [M.M.T.Rosa et al. 535] & ep & $\mathrm{F} 1$ & MA \\
\hline Microgramma vacciniifolia (Langsd. \& Fisch.) Copel. [D.M.Braz et al. 208] & ep & $\mathrm{F} 1$ & CE, MA \\
\hline Serpocaulon triseriale (Sw.) A. R. Sm. [E.L.Jacques et al. 1696] & er & $\mathrm{Aa}$ & $\mathrm{AM}, \mathrm{CA}, \mathrm{CE}, \mathrm{MA}, \mathrm{PA}, \mathrm{PN}$ \\
\hline \multicolumn{4}{|l|}{ PRIMULACEAE } \\
\hline Clavija spinosa (Vell.) Mez [M.M.T.Rosa et al. 540] & $a b$ & F1 & MA \\
\hline Jacquinia armillaris Jacq. [G.V.Somner et al. 1220] & av & Fl & AM, MA \\
\hline Myrsine parvifolia A.DC. [Observação local] & $a b$ & Af & CE, MA \\
\hline \multicolumn{4}{|l|}{ RHIZOPHORACEAE } \\
\hline Rhizophora mangle L. [Observação local] & av & $\mathrm{Mg}$ & AM, MA \\
\hline \multicolumn{4}{|l|}{ RUBIACEAE } \\
\hline Amaioua pilosa K.Schum. [M.M.T.Rosa et al. 537] & av & F1 & MA \\
\hline Borreria capitata (Ruiz \& Pav.) DC. [E.L.Jacques et al. 1683] & er & $\mathrm{Hv}$ & $\mathrm{AM}, \mathrm{CA}, \mathrm{CE}, \mathrm{MA}$ \\
\hline Borreria verticillata (L.) G. May [E.L.Jacques et al. 1716] & er & Нp & MA \\
\hline Chiococca alba (L.) Hitchc. [D.M.Braz et al. 210] & li & Fl & $\mathrm{AM}, \mathrm{CA}, \mathrm{CE}, \mathrm{MA}$ \\
\hline Coccocypselum capitatum (Graham) C.B.Costa \& Mamede [D.M.Braz et al. 254] & er & $\mathrm{Aa}$ & MA \\
\hline Coutarea hexandra (Jacq.) K. Schum [D.M.Braz et al. 205] & av & $\mathrm{F} 1$ & $\mathrm{AM}, \mathrm{CA}, \mathrm{CE}, \mathrm{MA}$ \\
\hline $\begin{array}{l}\text { Mitracarpus frigidus (Willd. ex Roem. \& Schult.) K.Schum. [E.L.Jacques et al. } \\
\text { 1700] }\end{array}$ & er & Aa & $\mathrm{AM}, \mathrm{CA}, \mathrm{CE}, \mathrm{MA}$ \\
\hline Randia armata (Sw.) DC. [D.M.Braz et al. 193] & av & $\mathrm{F} 1$ & $\mathrm{AM}, \mathrm{CA}, \mathrm{CE}, \mathrm{MA}$ \\
\hline Richardia brasiliensis Gomez [D.M.Braz et al. 240] & er & Hp & CA, CE, MA, PA \\
\hline Tocoyena bullata (Vell.) Mart. [E.L.Jacques et al. 1686] & $a b$ & $\mathrm{Aa}$ & $\mathrm{CA}, \mathrm{CE}, \mathrm{MA}$ \\
\hline \multicolumn{4}{|l|}{ RUTACEAE } \\
\hline $\begin{array}{l}\text { Conchocarpus heterophyllus (A. St.-Hil.) Kallunki \& Pirani [G.V.Somner et al. } \\
\text { 1439] }\end{array}$ & $\mathrm{ab}$ & Fl & $\mathrm{AM}, \mathrm{CA}, \mathrm{CE}, \mathrm{MA}$ \\
\hline Pilocarpus cf. riedelianus Engl. [D.M.Braz et al. 217] & av & $\mathrm{F} 1$ & MA \\
\hline Rauia nodosa (Engl.) Kallunki [D.M.Braz et al. 216] & av & $\mathrm{F} 1$ & MA \\
\hline \multicolumn{4}{|l|}{ SAPINDACEAE } \\
\hline Allophyllus puberulus (Cambess.) Radlk. [D.M.Braz et al. 209] & av & Fl & MA \\
\hline Cupania emarginata Cambess. [E.L.Jacques et al. 1703] & av & $\mathrm{Aa}$ & MA \\
\hline Matayba sp. [D.M.Braz et al. 203] & av & F1 & \\
\hline
\end{tabular}


Tabela 1. Continuação....

Table 1. Continued...

\begin{tabular}{|c|c|c|c|}
\hline $\begin{array}{c}\text { FAMÍLIA } \\
\text { Espécie (material testemunho, RBR) }\end{array}$ & Hábito & $\begin{array}{c}\text { Formação } \\
\text { vegetal }\end{array}$ & Distribuição \\
\hline Paullinia racemosa Wawra [D.M.Braz et al. 196] & av & $\mathrm{Fl}$ & MA \\
\hline Paullinia weinmaniifolia Mart. [D.M.Braz et al. 207] & li & $\mathrm{Aa} ; \mathrm{Fl}$ & MA \\
\hline Serjania salzmanniana Schltdl. [M.M.T.Rosa et al. 515] & li & $\mathrm{Fl}$ & $\mathrm{AM}, \mathrm{CE}, \mathrm{MA}$ \\
\hline Urvillea rufescens Cambess. [D.M.Braz et al. 206] & li & $\mathrm{Fl}$ & MA \\
\hline Urvillea triphylla (Vell.) Radlk. [D.M.Braz et al. 211] & li & $\mathrm{Fl}$ & MA \\
\hline SAPOTACEAE & & & \\
\hline Manilkara salzmannii (A. DC.) Lam. [E.L.Jacques et al. 1705] & $\mathrm{ab}$ & $\mathrm{Fl} ; \mathrm{Aa}$ & MA \\
\hline SCHIZAEACEAE & & & \\
\hline $\begin{array}{l}\text { Actinostachys pennula (Sw.) Hook. [M.M.T.Rosa et al. 529] } \\
\text { SMILACACEAE }\end{array}$ & er & $\mathrm{Fl}$ & $\mathrm{AM}, \mathrm{MA}$ \\
\hline $\begin{array}{l}\text { Smilax sp.2 [M.M.T. Rosa et al. 539] } \\
\text { SOLANACEAE }\end{array}$ & li & $\mathrm{Fl}$ & \\
\hline Cestrum axillare Vell. [G.V.Somner et al. 1212] & $\mathrm{ab}$ & $\mathrm{Fl}$ & $\mathrm{AM}, \mathrm{CA}, \mathrm{CE}, \mathrm{MA}, \mathrm{PN}$ \\
\hline Solanum torvum Sw. [D.M.Braz et al. 185] & $a b$ & $\mathrm{Fl}$ & MA \\
\hline Solanum insidiosum Mart. [G.V.Somner et al. 1433] & av & $\mathrm{Fl}$ & MA \\
\hline $\begin{array}{l}\text { Solanum jussiaei Dunal [G.V.Somner et al. 1210] } \\
\text { TYPHACEAE }\end{array}$ & $a b$ & $\mathrm{Fl}$ & MA \\
\hline $\begin{array}{l}\text { Typha domingensis Pers. [M.M.T. Rosa et al. 543] } \\
\text { VERBENACEAE }\end{array}$ & er & $\mathrm{Hi}$ & $\mathrm{AM}, \mathrm{CA}, \mathrm{CE}, \mathrm{MA}, \mathrm{PA}, \mathrm{PN}$ \\
\hline Lantana fucata Lindl. [D.M.Braz et al. 190] & $\mathrm{ab}$ & $\mathrm{Fl}$ & $\mathrm{CA}, \mathrm{CE}, \mathrm{MA}$ \\
\hline $\begin{array}{l}\text { Stachytarpheta crassifolia Schauer [Observação local] } \\
\text { VIOLACEAE }\end{array}$ & er & Aa & $\mathrm{CA}, \mathrm{CE}, \mathrm{MA}$ \\
\hline Anchietea pyrifolia (Mart.) G.Don [D.M.Braz et al. 192] & li & $\mathrm{Fl}$ & $\mathrm{CA}, \mathrm{CE}, \mathrm{MA}, \mathrm{PA}, \mathrm{PN}$ \\
\hline $\begin{array}{l}\text { Hybanthus calceolaria (L.) Oken [A.V.S.Couto et al. s/n] } \\
\text { VITACEAE }\end{array}$ & er & $\mathrm{Fl}$ & $\mathrm{AM}, \mathrm{CA}, \mathrm{CE}, \mathrm{MA}, \mathrm{PN}$ \\
\hline Cissus verticillata (L.) Nicolsoni \& C.E.Jarvis [M.M.T.Rosa et al. 544] & li & Hi & $\mathrm{AM}, \mathrm{CA}, \mathrm{CE}, \mathrm{MA}, \mathrm{PA}, \mathrm{PN}$ \\
\hline
\end{tabular}

As formações vegetais das restingas brasileiras ainda não estão bem delimitadas, pois há deficiência de dados florísticos, estruturais e ambientais de muitos trechos do litoral (Araújo \& Henriques 1984, Menezes \& Araújo 2005). Entretanto, com base na heterogeneidade florística observada nas diferentes formações de restingas, Magnago et al. (2011) confirmam sua estruturação em formações distintas e, em geral, bem delimitadas. Considerando as restingas do sudeste brasileiros, bastante estudadas ultimamente, ainda que com fortes semelhanças, cuidados devem ser tomados ao correlacionar as formações vegetais, mesmo que próximas geograficamente. Araújo et al. (2009) constataram essa necessidade ao verificarem que pode haver forte correspondência nas formações sem, contudo, haver relação na composição das espécies. Isso foi comprovado nos estudos de Magnano et al. (2011) com relação às restingas do Rio de Janeiro e do Espírito Santo, onde os autores verificaram semelhanças fisionômicas, mas com pouca correspondência na composição das espécies. Exceção é feita para a formação halófila-psamófila, onde ocorrem espécies em comum nas restingas de norte a sul do país. Já Sacramento et al. (2007) verificaram a semelhança entre as espécies e diferenças no arranjo fisionômico entre duas restingas próximas.

Foi encontrada considerável relação das formações vegetais identificadas na restinga de Praia das Neves com as formações propostas para as restingas do Rio de Janeiro (Menezes \& Araújo 2005), tanto nos aspectos físicos como na composição florística. Entretanto, não foram verificadas as seguintes formações: Floresta inundável, Floresta inundada, Arbustiva aberta inundável e Arbustiva fechada de cordão arenoso. Por outro lado, em outros estudos realizados em restingas mais próximas do Estado do Rio de Janeiro (Araújo et al. 2009), há menos semelhança na composição das espécies.

Uma formação denominada Arbustiva fechada de cordão arenoso por Menezes \& Araújo (2005) é denominada por outros autores como "thicket de Myrtaceae" (Araújo \& Henriques 1984), e "thicket fechado de Myrtaceae" (Araújo 1992). Essa formação não foi observada na Praia das Neves, da mesma forma como nas restingas de Massambaba, RJ (Araújo et al. 2009), e em Vila Velha, ES (Magnago et al. 2007). Igualmente, nas restingas do nordeste esse tipo de formação não é observado (Pinto et al. 1984, Almeida Junior et al. 2009, Castro et al. 2012). A ausência das formações florestais inundável e inundada e Arbustiva aberta inundável na restinga de Praia das Neves pode ser explicado pelas condições da declividade local, quase nula, e também da devastação a que a floresta e outras fitofisionomias foram submetidas.

Dentre as formações da restinga de Praia das Neves, merecem destaque: a vegetação arbustiva aberta, que ocupa as maiores extensões; a vegetação florestal que, embora fragmentada, ainda apresenta alguns trechos bem preservados; e a formação arbustiva fechada de pós-praia que é a mais depauperada, reduzida atualmente a raros fragmentos.

\section{Florística}

Registramos 167 espécies da flora, reunidas em 58 famílias botânicas, distribuídas nas diferentes formações da restinga de Praia das Neves e vegetações associadas (Tabela 1). As famílias 
com maior riqueza foram: Fabaceae (16), Poaceae (12), Rubiaceae (10), Sapindaceae (oito), Bromeliaceae (oito), Asteraceae (seis), Acanthaceae (cinco) e Bignoniaceae (cinco).

As mesmas famílias são, em geral, indicadas como as mais representativas para outras restingas do Estado Espírito Santo (Pereira \& Assis 2000, Assis et al. 2004) e também do Rio de Janeiro (Araújo \& Henriques 1984, Menezes \& Araújo 2005, Araújo et al. 2009). Igualmente, para uma restinga do nordeste do Brasil, Almeida Junior et al. (2009) apontaram praticamente as mesmas famílias como as de maior riqueza. As Acanthaceae, embora não representativas nas restingas fluminenses ou capixabas até então, apareceram como a quarta família mais rica no estrato herbáceo da restinga de Bertioga, SP (Martins et al. 2008).

Analisando a distribuição das espécies levantadas (Tabela 1), cerca de $20 \%$ ocorrem nos quatro principais biomas brasileiros (Amazônia, Caatinga, Cerrado e Floresta Atlântica), enquanto que cerca de 30\% são exclusivas da Floresta Atlântica. Dentre essas últimas, inúmeros táxons amostrados têm distribuição restrita às restingas, tais como Cupania emarginata, Chrysobalanus icaco, Erythroxylum ovalifolium, Paullinia weinmaniifolia e Peplonia asteria. Outros, como Actinocephalus ramosus, Cereus fernambucensis e Gaylussacia brasiliensis, tem distribuição disjunta nas restingas e em afloramentos rochosos mais para o interior do país. Destaca-se ainda a ocorrência de Aechmea saxicola, restrita ao Espírito Santo e Rio de Janeiro (Forzza et al. 2010), Neoregelia cruenta, que foi praticamente erradicada das restingas da cidade do Rio de Janeiro, pela ação de exploradores (Leme \& Marigo 1993) e Aechmea blanchetiana, uma das espécies mais comuns na restinga de Praia das Neves, onde forma touceiras; essa espécie pode também ocorrer na Floresta Ombrófila Densa, como epífita (Leme \& Marigo 1993, Stehmann et al. 2009). A maioria das espécies se mostrou exclusiva de uma única formação, entretanto, algumas, como Allagoptera arenaria (guriri-da-praia), Cereus fernambucensis e Stylosanthes guianensis, foram registradas em diferentes formações.

Salienta-se que inventários mais detalhados na restinga de Praia das Neves poderão apontar novas espécies. Considerando alguns estudos de inventários florísticos sistematizados em restinga que incluem os diferentes hábitos vegetais, Araújo et al. (2009) levantaram 664 espécies vegetais para uma área de 76.300 ha na APA de Massambaba (Saquarema, Araruama e Arraial do Cabo), RJ, e para o Espírito Santo, nas restingas de Camburi foram amostradas 211 espécies (Pereira \& Assis 2000) e em Conceição da Barra 415 (Pereira \& Gomes 1993), em 200 ha e 1031 ha, respectivamente. Já para o nordeste e norte brasileiros, usando como exemplo uma área de cerca de 11.200 ha de restinga no Ceará, foram registradas 382 espécies (Castro et al. 2012), e para todo o estado do Pará, Silva et al. (2010) somaram 411 espécies. Tais números sugerem diferenças na riqueza de espécies, assim como nas formações encontradas em restingas de diferentes regiões do Brasil.

\section{Conservação da restinga}

A riqueza paisagística e biológica é constatada com base nas diferentes fitofisionomias (formações vegetais) que compõe a restinga de Praia das Neves, típicas desse ecossistema litorâneo. Seu bom estado de conservação é ainda atestado pelo registro das espécies Jacquinia armillaris (Figura 6d), Justicia cydoniifolia (Figura 9a) e Schaneria lophura (Figura 9b), indicadas como vulneráveis na listagem das espécies ameaçadas no Estado do Espírito Santo (Simonelli \& Fraga 2007). Em relação à primeira, essa é a espécie dominante na formação arbustiva fechada de pós-praia e que margeia a principal área ocupada nas praias, favorecendo a extinção da formação e explicando o status de vulnerabilidade da espécie. De fato, essa está limitada a pequenos trechos remanescentes na restinga de Praia das Neves (Figuras 2 e 3c). Outros elementos da biota local são dependentes da vegetação da restinga, como a largatixa-de-areia, Liolaemus lutzae (Soares \& Araujo 2008), espécie igualmente ameaçada de extinção (Instituto... 2003). Esses são aspectos de interesse para estudos futuros e corroboram a necessidade de preservação desses ambientes litorâneos, que são altamente suscetíveis à expansão urbana.

Parte da vegetação original da restinga de Praia das Neves foi devastada, submetida a loteamentos (Figura 10a) e outros usos. A imagem da Praia das Neves em vista do satélite Landsat na figura de Dias \& Silva (1984) (Figura 8) denota que a floresta ocupava maiores extensões e é possível que originalmente formasse uma faixa contínua, relativamente larga, paralela à costa. Atualmente,
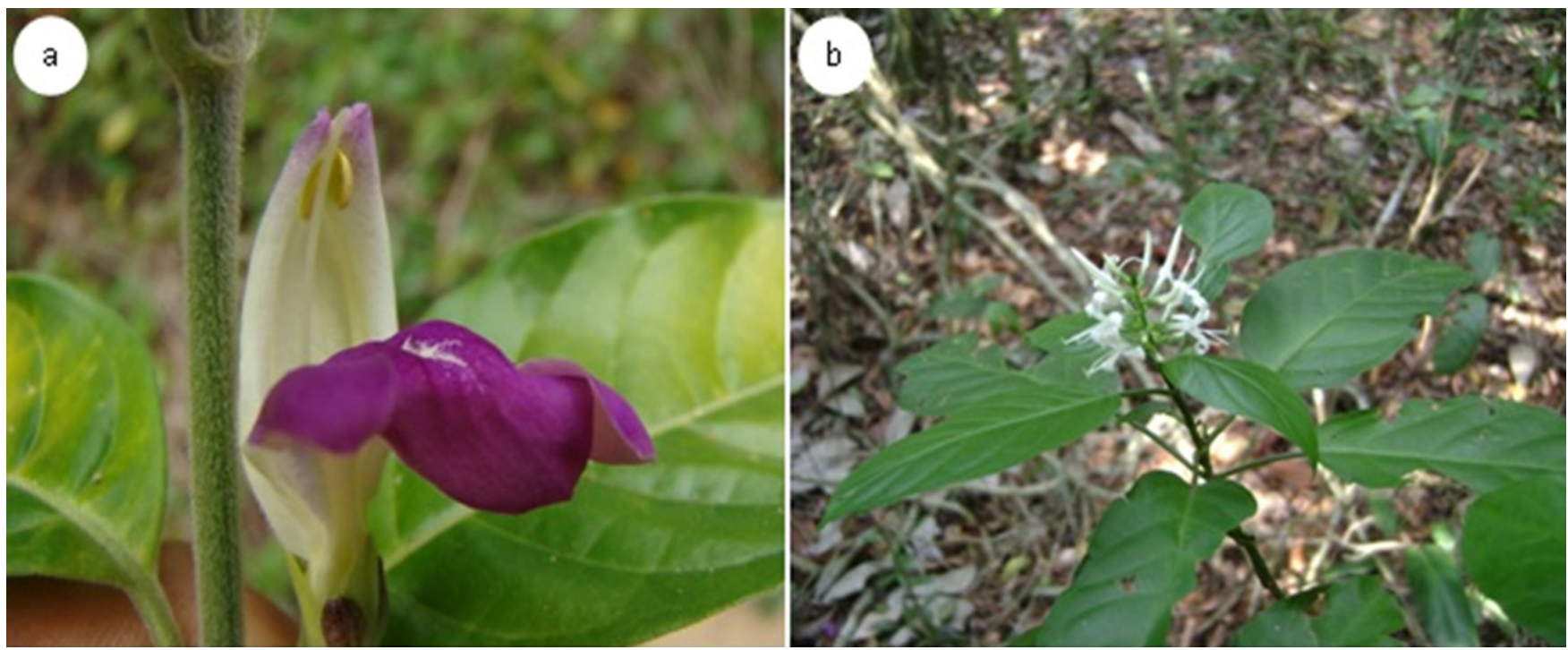

Figura 9. Espécies ameaçadas da flora do Estado do Espírito Santo registradas na restinga de Praia das Neves. a) Justicia cydoniifolia na Floresta de cordão arenoso. b) Schaueria lophura na Floresta de cordão arenoso.

Figure 9. Threatened species of the flora of Espírito Santo State registered in the sandy coastal plain (restinga) of "Praia das Neves", ES, Brazil. a) Justicia cydoniifolia in the Ridge forest. b) Schaueria lophura in the Ridge forest. 


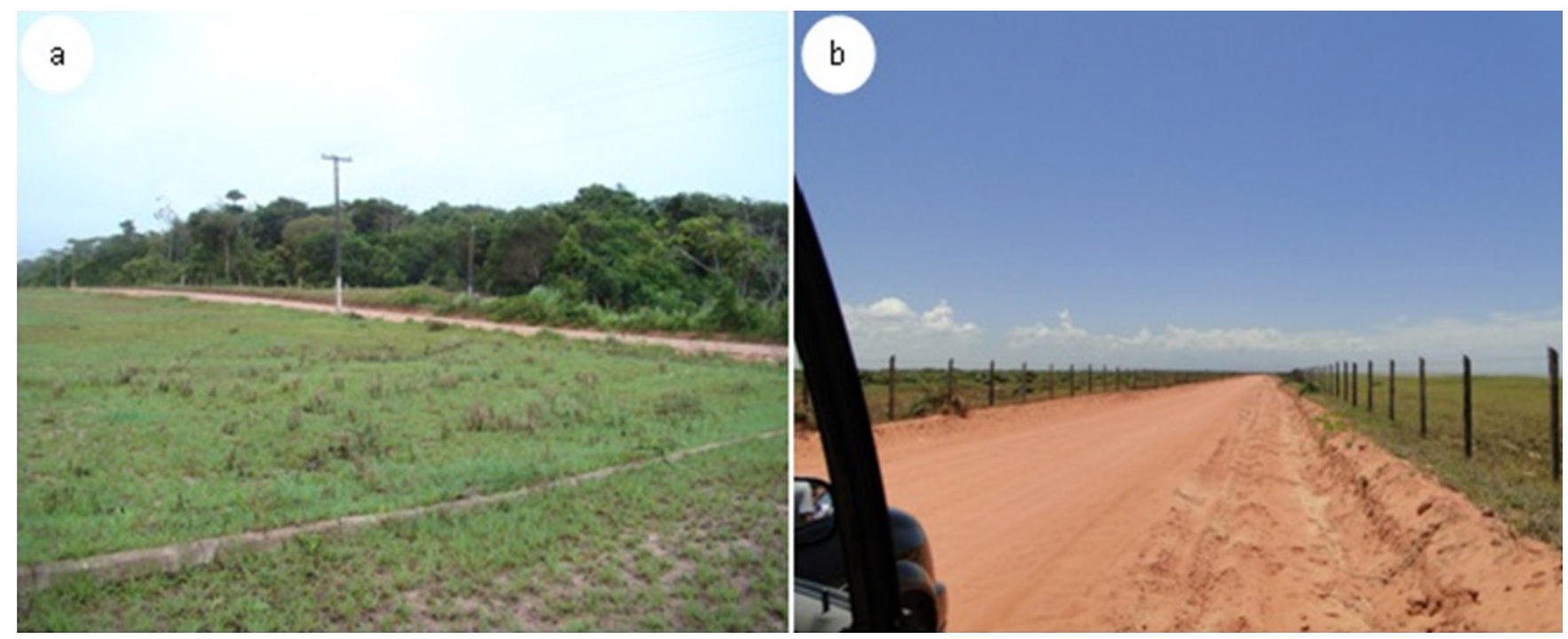

Figura 10. Restinga de Praia das Neves, ES. a) Floresta ao fundo, margeada por loteamento. b) Cerca limitando o acesso à praia e as demais áreas da restinga.

Figure 10. Sandy coastal plain (restinga) of "Praia das Neves", ES, Brazil. a) Forest in the background, bordered by blending. b) Fence limiting the access to the beach and other areas of the sandy coastal plains.

a vegetação arbustiva densa de pós-praia e a floresta ocorrem em fragmentos ao longo da costa. Apesar do desmatamento a que foi submetida, essa restinga permanece com extensos trechos em bom estado de conservação e com remanescentes importantes de formações vegetais costeiras no Espírito Santo. Recentemente, a maior extensão da restinga de Praia das Neves foi delimitada com a intensão de construção de um porto para exportação de ferro, segundo informação popular (Figura 10b).

Considerando a riqueza em formações vegetais típicas das restingas, da flora e da fauna associadas, a restinga de Praia das Neves se enquadra nas Áreas de Preservação Permanente, descritas e legitimadas na legislação federal brasileira (Resolução CONAMA no 303, de 20 de março de 2002 (Conselho... 2002); Novo Código Florestal, Lei no 12.651 de 25 de maio de 2012 (Brasil... 2012)). Ações futuras deveriam incluir a proteção da área ou parte dela como Unidade de Conservação, assegurando a preservação da restinga local, com suas diferentes formações e a biota associada. Essa proposta corrobora com a de Rocha et al. (2003) que, com base na elevada diversidade biológica e seu bom estado de conservação, recomenda a criação de Unidade de Conservação, preferencialmente de Proteção Integral para a restinga de Praia das Neves.

\section{Agradecimentos}

Os autores agradecem ao Fundo Nacional do Meio Ambiente (FNMA) e ao Prof. Alexandre Fernandes Bamberg de Araújo pelo apoio financeiro através do Projeto "Manejo da lagartixa-de-areia, Liolaemus lutzae"; a Karina Marques, Luiz Marcelo Fiuza, Thiago Barbosa e Fernando Antônio S. Pinto pela ajuda e participação nas atividades de campo; a Leandro Guccione pela confecção do mapa de vegetação; aos taxonomistas Marcelo da Costa Souza pelo auxílio na identificação das Myrtaceae, Marcos Alberto Nadruz Coelho pelas Araceae, Jorge Pereira Fontella pelas Apocynaceae, Robson Daumas Ribeiro (in memoriam) pelas Fabaceae, Christian da Silva pelas Poaceae, Bruno C. Bandeira pelas Solanaceae, Felipe Fajardo V. A. Barberena pelas Orchidaceae e Jefferson Guedes C. Sobrinho pelas Malvaceae-Pseudobombax.

\section{Referências Bibliográficas}

ALMEIDA JUNIOR, E.B., OLIVO, M.A., ARAÚJO, E.L. \& ZICKEL, C.S. 2009. Caracterização da vegetação de restinga da RPPN de Maracaípe, $\mathrm{PE}$, Brasil, com base na fisionomia, flora, nutrientes do solo e lençol feático. Acta Bot. Bras. 23(1):36-48. http://dx.doi.org/10.1590/S010233062009000100005

AMARAL, D.D., PROST, M.T., BASTOS, M.N.C., COSTA NETO, S.V. \& SANTOS, J.U.M. 2008. Restingas do litoral amazônico, estados do Pará e Amapá, Brasil. Bol. Mus. Para. Emílio Goeldi. 3(1):35-67.

ANGIOSPERM PHYLOGENY GROUP - APG. 2009. An update of the Angiosperm Phylogeny Group classification for the orders and families of flowering plants: APG III. Bot. j. Linn. Soc. 161:105-121. http://dx.doi. org/10.1111/j.1095-8339.2009.00996.x

ARAÚJO, D.S.D. 1992. Vegetation types of Sandy coastal plains of tropical Brazil: a first approximation. In Coastal Plant Communities of Latin America (U. Seeliger, ed.). Academic Press, New York, p.337-347.

ARAÚJO, D.S.D. \& HENRIQUES, R.P.B. 1984. Análise florística das restingas do Estado do Rio de Janeiro. In Restingas: Origem, Estrutura, Processos (L.D. Lacerda, D.S.D. Araújo, R. Cerqueira \& B. Turcq, orgs.). CEUFF, Niterói, p.159-193.

ARAÚJO, D.S.D., SÁ, C.F.C., PEREIRA, J.F., GARCIA, D.S., FERREIRA, M.V., PAIXÃO, R.J., SCHENEIDER, S.M. \& FONSECA-KRUEL, V.S. 2009. Área de Proteção Ambiental de Massambaba, Rio de Janeiro: caracterização fitofisionômica e florística. Rodriguésia 60(1):67-96.

ASSIS, A. M., THOMAZ, L. D. \& PEREIRA, O. J. 2004. Florística de um trecho de floresta de restinga no Município de Guarapari, Espírito Santo, Brasil. Acta Bot. Bras. 18(1):191-201. http://dx.doi.org/10.1590/S010233062004000100016

BRASIL. 2012. Novo Código Florestal, Lei no 12.651 de 25 de maio de 2012. http://www.planalto.gov.br/ccivil_03/_Ato2011-2014/2012/Lei/L12651. htm. (último acesso em 25/06/2013).

CASTRO, A.S.F., MORO, M.F. \& MENEZES, M.O.T. 2012. O complexo vegetacional da Zona Litorânea no Ceará: Pecém, São Gonçalo do Amarante. Acta Bot. Bras. 26(1): 108-124. http://dx.doi.org/10.1590/ S0102-33062012000100013 
CONSELHO NACIONAL DO MEIO AMBIENTE - CONAMA. 2002. Resolução $\mathrm{n}^{\circ}$ 303, de 20 de março de 2002. http://www.planalto.gov. br/ccivil_03/_Ato2011-2014/2012/Lei/L12651.htm (último acesso em 26/06/2013).

DIAS, G.T.M. \& SILVA, C.G. 1984. Geologia de depósitos arenosos costeiros emersos - exemplos ao longo do litoral fluminense. In Restingas: Origem, Estrutura, Processos (L.D. Lacerda, D.S.D. Araújo, R. Cerqueira \& B. Turcq, orgs.). CEUFF, Niterói, p.47-60.

FORZZA, R. C., BAUMGRATZ, J.F.A., BICUDO, C.E.M., CARVALHO JR., A.A, COSTA, A., COSTA, D.P., HOPKINS, M., LEITMAN, P.M., LOHMANN, L.G., MAIA, L.C., MARTINELLI, G., MENEZES, MORIM, M.P. NADRUZ COELHO, M.A., PEIXOTO,A.L., PIRANI, J.R. PRADO, J., QUEIROZ, L.P., V.C. SOUZA, STEHMANN, J.R., SYLVESTRE, L.S., WALTER, B.M.T., ZAPPI, D. (Orgs.). 2010. Catálogo de plantas e fungos do Brasil. Rio de Janeiro, Andrea Jakobsson Estúdio, Jardim Botânico do Rio de Janeiro. 2 v.

GOOGLE EARTH. http://www.google.com/earth/index.html (último acesso em 09/09/2012)

INSTITUTO BRASILEIRO DO MEIO AMBIENTE E DOS RECURSOS NATURAIS RENOVÁVEIS - IBAMA. 2003. Lista da fauna silvestre brasileira ameaçada de extinção. http://www.ibama.gov.br (último acesso em 20/01/2012).

INSTITUTO BRASILEIRO DE GEOGRAFIA E ESTATÍSTICA - IBGE. 2012. http://www.ibge.gov.br (último acesso em 30/03/2012).

INTERNATIONAL PLANT NAME INDEX - IPNI. 2012. http://www.ipni. org (último acesso em 07/09/2012).

JARDIM BOTÂNICO DO RIO DE JANEIRO. Lista de Espécies da Flora do Brasil. http://reflora.jbrj.gov.br/jabot/listaBrasil/ConsultaPublicaUC/ ConsultaPublicaUC.do (último acesso em 17/02/2013).

LAMEGO, A.R. 1946. O homem e a restinga. Lidador, Rio de Janeiro.

LEME, E.M.C. \& MARIGO, L.C. 1993. Bromélias na natureza. Marigo Comunicação Visual, Rio de Janeiro.

MARTINS, S.E., ROSSI, L., SAMPAIO, P.S.P. \& MAGENTA, M.A.G. 2008. Caracterização florística de comunidades vegetais de restinga em Bertioga, SP, Brasil. Acta Bot. Bras. 22(1):249-274. http://dx.doi. org/10.1590/S0102-33062008000100024

MAGNAGO, L.F.S., PEREIRA, O.J., MATOS, F.A.R. \& SOUZA, P.F. 2007. Caracterização Fitofisionômica da Restinga na Morada do Sol, Vila Velha, ES. Rev. Bras. Biociênc. 5(1):456-458.

MAGNAGO, L.F.S., MARTINS, S.V. \& PEREIRA, O.J. 2011. Heterogeneidade florística das fitocenoses de restingas nos estados do Rio de Janeiro e Espírito Santo, Brasil. Rev. Arvore 35(2):245-254. http://dx.doi. org/10.1590/S0100-67622011000200009

MENEZES, L.F.T. \& ARAÚJO, D.S.D. 2005. Formações vegetais da restinga da Marambaia, Rio de Janeiro. In História Natural da Marambaia (L.F.T. Menezes, A.L. Peixoto \& D.S.D. Araújo). EDUR, Seropédica, p.67-132.

MONTEZUMA, R.C.M. \& ARAÚJO, D.S.D. 2007. Estrutura da vegetação de uma restinga arbustiva inundável no Parque Nacional da Restinga de Jurubatiba, Rio de Janeiro. Pesq. Bot. 58:157-176.

MUEHE, D. 1984. Evidências de recuo dos cordões litorâneos em direção ao continente no litoral do Rio de Janeiro. In Restingas: Origem, Estrutura, Processos (L.D. Lacerda, D.S.D. Araújo, R. Cerqueira \& B. Turcq, orgs.). CEUFF, Niterói, p.75-80.

PEEL, M.C., FINLAYSON, B.L. \& MCMAHON, T.A. 2007. Updated world map of the Köppen-Geiger climate classification. Hydrol. Earth Syst. Sci. 11:1633-1644. http://dx.doi.org/10.5194/hess-11-1633-2007
PEREIRA, M.C.A., CORDEIRO, S.C. \& ARAÚJO, D.S. D. 2004. Estrutura estrato herbáceo na formação aberta de Clusia do Parque Nacional da Restinga de Jurubatiba, RJ, Brasil. Acta. Bot. Bras. 18(3):677-687. http:// dx.doi.org/10.1590/S0102-33062004000300025

PEREIRA, O.J. 2003. Restinga: origem, estrutura e diversidade. In Desafios da botânica brasileira no novo milênio: inventário, sistematização e conservação da biodiversidade vegetal (M.A.G. Jardim, M.N.C. Bastos \& J.U.M. Santos, orgs.). Embrapa, Museu Paraense Emílio Goeldi, p.177-179.

PEREIRA, O.J. \& ASSIS, A.M. 2000. Florística da restinga de Camburi, Vitória, ES. Acta Bot. Bras. 14(1):99-111.

PEREIRA, O.J. \& GOMES, J.M.L. 1993. Levantamento florístico das comunidades vegetais de restinga no Município de Conceição da Barra, ES. In III Simpósio de Ecossistemas da costa brasileira. ACIESP, p.67-78.

PEREIRA, O.J., THOMAZ, L.D. \& ARAÚJO, D.S.D. 1992. Fitossociologia da vegetação de ante dunas da restinga de Setiba/Gurapari e em Interlagos/ Vila Velha, ES. Bol. Mus. Biol. Mello Leitão 1:65-75.

PINTO, G.C.P., BAUTISTA, H.P. \& FERREIRA, J.A.C.A. 1984. A restinga do litoral nordeste do Estado da Bahia. In Restingas: Origem, Estrutura, Processos (L.D. Lacerda, D.S.D. Araújo, R. Cerqueira \& B. Turcq, orgs.). CEUFF, Niterói, p.195-216.

ROCHA, C.F.D., BERGALLO, H.G., ALVES, M.A.S. \& SLUYS, M.V. 2003. A Biodiversidade nos Grandes remanescentes florestais do Estado do Rio de Janeiro e nas restingas da Mata Atlântica. Rima, São Carlos.

SACRAMENTO, A.C., ZICKEL, C.S. \& ALMEIRA JUNIOR, E.B. 2007 Aspectos florísticos da vegetação de restinga no litoral de Pernambuco. Rev. Arvore 31(6):1121-1130. http://dx.doi.org/10.1590/S010067622007000600017

SAMPAIO, D. 2005. Restinga. In Árvores da restinga: Guia de identificação (D.Sampaio, V.C. Souza, A.A. Oliveira, J.Paula-Souza \& R.R. Rodrigues) Neotrópica, São Paulo, p.25-30.

SILVA, J.G. \& SOMNNER, G.V. 1984. A vegetação de restinga na Barra de Maricá. In Restingas: Origem, Estrutura, Processos (L.D. Lacerda, D.S.D. Araújo, R. Cerqueira \& B. Turcq, orgs.). CEUFF, Niterói, p.217-225.

SILVA, R.M., MEHLIG, U., SANTOS, J.U.M. \& MENEZES, M.P.M. 2010. The coastal restinga vegetation of Pará, Brazilian Amazon: a synthesis. Rev. Brasil. Bot. 33(4):563-573.

SIMONELLI, M. \& FRAGA, C.N. (Orgs.). 2007. Espécies da flora ameaçadas de extinção no estado do Espírito Santo. Ipema, Vitória.

SMITH, A.R., PRYER, K.M., SCHUETTPELZ, E., KORALL, P., SCHNEIDER, H. \& WOLF, P.G. 2006. A classification of extant ferns. Taxon 55 (3):705-731. http://dx.doi.org/10.2307/25065646

SOARES, A.H.B. \& ARAUJO, A.F.B. 2008. Experimental introduction of Liolaemus lutzae (Squamata: Iguanidae) in Praia das Neves, State of Espírito Santo, Brazil: a descriptive study 18 years later. Rev. Brasil. Zool. 25(4):640-646

STEHMANN, J.R., FORZZA, R.C., SALINO, A., SOBRAL, M., COSTA, D.P. \& KAMINO, L.H.Y. 2009. Plantas da Floresta Atlântica. Jardim Botânico do Rio de Janeiro, Rio de Janeiro.

THIERS, B. 2013. Index Herbariorum: A global directory of public herbaria and associated staff. New York Botanical Garden's Virtual Herbarium. http://sweetgum.nybg.org/ih (último acesso em 11/10/2012).

THOMAZ, L. D. \& MONTEIRO, R. 1993. Distribuição de espécies na comunidade halófila-psamófila ao longo do litoral do Estado do Espírito Santo. Arq. Biol. Tecnol. 36(2):375-399. 